\title{
Curves of low positive genus and low degree on a general heptic hypersurface of $\mathbb{P}^{5}$
}

\author{
E. Ballico*
}

\begin{abstract}
We study the non-existence of smooth curves of low degree and low positive genus on a general heptic hypersurface of $\mathbb{P}^{5}$. The genus 0 case was proved for the same degrees by Hana - Johnsen and Cotterill.
\end{abstract}

\section{Introduction}

H. Clemens conjectured that for any degree $d>0$ a general quintic hypersurface of $\mathbb{P}^{4}$ contains only finitely many smooth rational curves of degree $d$; the stronger form of the conjecture says that the same is true for singular rational curves, except rational degree 5 plane curves ([5], [6], [9], [19], [20], [21], [22], [30]). There are results on other Fano 3-folds ([21],[26, Theorem 2], [22, Theorem 1.2]) results for higher genera ([23], [24]) and results on higher dimensional hypersurfaces of general types ([32]). In particular for every integer $k \geq 8$ for a very general degree $k$ hypersurface $X \subset \mathbb{P}^{5}$ and any integral curve $D \subset X$, we have $\operatorname{deg}(D) \leq 2 \widetilde{g}-2$, where $\widetilde{g}$ is the geometric genus of $D$ ([31], [32, Theorem 3.9]). The case $k=7$, i.e. the case of a general heptic hypersurface of $\mathbb{P}^{5}$, was singled out as an interesting boundary case ([7], [14]). A general heptic hypersurface contains exactly 698005 lines and no rational curve (not even singular ones) with degree $d$ if $2 \leq d \leq 16$ ([7], [14], [28, Theorem 1.1]). There are two main tools available for rational curves, but not for smooth curves of higher genus (a very strong result on the strata by splitting type of the restricted tangent bundle and

\footnotetext{
*The author was partially supported by MIUR and GNSAGA of Indam (Italy)

Received by the editors in December 2015 - In revised form in March 2016.

Communicated by J. Fine.

2010 Mathematics Subject Classification : 14J32; 14M10; 14H50.

Key words and phrases : heptic hypersurface; general hypersurface; curves in a hypersurface.
} 
the use of semigroups related to a rational parametrization). See [6] and [7] for a full use of these tools and [8] for a full use of the latter tool in another context.

For any set $S \subseteq \mathbb{P}^{5}$ let $\langle S\rangle$ denote its linear span. In this paper we prove the following result.

Theorem 1. Let $W \subset \mathbb{P}^{5}$ be a general heptic hypersurface. For all integers $d, g$ with $d \leq 16$ and $1 \leq g \leq 3 \mathrm{~W}$ does not contain any smooth curve $C$ of genus $g$ and degree $d$ with $\operatorname{dim}(\langle C\rangle) \neq 3$.

Only numerical reasons prevent us to get degrees $d$ a little bit higher or genera $g$ a little bit higher or to cover the curves spanning a 3-dimensional linear subspace. We prove the following result, but several parts of its proof works for higher genera and/or higher degree.

Proposition 1. A general heptic hypersurface of $\mathbb{P}^{5}$ contains no smooth curve $C$ of genus 1 and degree $d \leq 14$.

In section 2 (resp. section 3, resp. section 4) we prove the part of Theorem 1 concerning curves $C$ spanning a linear subspace $\langle C\rangle$ of $\mathbb{P}^{5}$ of dimension $\leq 2$ (resp. 5, resp. 4). In section 5 we give a few results for the case $\operatorname{dim}(\langle C\rangle)=3$ and prove Proposition 1. Section 4 contains more lemmas than the ones needed to prove Theorem 1 and even so in many places we know how to improve the lemmas by 1 . Anyway, section 3 does not work if $(d, g) \in\{(17,0),(17,1)\}$, the next interesting cases.

Since for any smooth hypersurface $W \subset \mathbb{P}^{n}, n \geq 4$, with $\operatorname{deg}(W) \geq 2$ there is a codimension 2 subvariety $Z \subset W$ which is not the intersection of $W$ and a codimension 2 subvariety of $\mathbb{P}^{n}$ ([29], [25]), there are plenty of curves on a very general heptic hypersurface $X$, which are not easily described, but certainly not unexpected. A dimensional count suggests that a very general heptic hypersurface has no curve with very low genus. If we take curves with arithmetic genus $q$ and degree $d \gg q$, then the dimensional count is better for high $q$ than for $q=0$ (when $d \gg q$ the dimensional count is even better for degenerate curves than for non-degenerate ones).

Question 1. Let $X \subset \mathbb{P}^{5}$ be a very general heptic hypersurface. Is it true that $X$ contains no elliptic curve? Is it true that for each $q \in \mathbb{N}$ there is an integer $d(q)$ such that $X$ has no curve of arithmetic genus $q$ and degree $d>d(q)$ ?

Our tools cannot solve these questions (at the very least we need $7 d+1-q<$ $\left.\left(\begin{array}{c}12 \\ 5\end{array}\right)\right)$, but we made an attempt to see where not to find counterexamples to this kind of questions. A strong feature of [7] (and also of [6] and [8]) is that it works for singular rational curves and this is the best way to state problems related to Clemens' conjecture as non-existence or finiteness results for maps from moduli schemes of curves to a varying target, e.g. a very general heptic hypersurface, i.e. in the set-up of Kontsevich moduli spaces with a varying target. Unfortunately, our tools use the arithmetic genus of the image and so at most we may recover (for low degrees) singular curves with low arithmetic genus. Theorem 1 and Proposition 1 are stated only for smooth curves, because in the singular case (but with arithmetic genus $q \leq 3$ ) we can handle only lower degrees. The first lemmas 
to be generalized for the interested reader are Lemmas 2 and 3, because a nondegenerate singular curve $C \subset \mathbb{P}^{5}$ has hyperplane sections $C \cap H$ which are not curvilinear. Moreover, if $q=1$ the singular curves are rational and so they do not exist for $d \leq 16$ on a very general heptic by [7]. If $q=2,3$ knowing by [7] the case of rational curves we may say that the non-rational ones have very mild singularities, and it helps, even for the generalizations of Lemmas 2 and 3.

Thanks are due to a referee for useful remarks.

\section{Preliminaries}

We work over the complex number field.

Let $M_{d, g}$ denote the set of all smooth curves $C \subset \mathbb{P}^{5}$ with degree $d$, genus $g$ and $h^{1}\left(\mathcal{O}_{C}(1)\right)=0$. The algebraic set $M_{d, g}$ is irreducible, because we only took nonspecial line bundles for the embeddings. Fix $C \in M_{d, g}$ and let $N_{C}$ be the normal bundle of $C$ in $\mathbb{P}^{5}$. Since $\operatorname{dim}(C)=1$, we have $h^{2}(\mathcal{F})=0$ for every coherent sheaf $\mathcal{F}$ on $C$. Therefore for any surjection $\mathcal{G} \rightarrow \mathcal{E}$ of coherent sheaves on $C$, the associated map $H^{1}(\mathcal{G}) \rightarrow H^{1}(\mathcal{E})$ is surjective. Hence $h^{1}(\mathcal{E})=0$ if $h^{1}(\mathcal{G})=0$. The Euler's sequence shows that $T \mathbb{P}^{5}$ is a quotient of $\mathcal{O}_{\mathbb{P}^{5}}(1)^{\oplus 6}$. Hence $h^{1}\left(N_{C}\right)=0$. Thus $M_{d, g}$ is smooth and of dimension $h^{0}\left(N_{C}\right)=6 d+2-2 g$.

If we drop the condition $h^{1}\left(\mathcal{O}_{C}(1)\right)=0$, then when $g \leq 3$ we only need to add the case $(d, g)=(4,3)$ (canonically embedded non-hyperelliptic curves of genus 3 ). See Remark 2 for a proof that a general heptic hypersurface contains no canonically embedded curve of genus 3 .

For any integer $r \geq 1$ let $M_{d, g}(r)$ be the set of all $C \in M_{d, g}$ whose linear span has dimension $r$. Since $g>0$, we have $M_{d, g}(1)=\varnothing$. Since $1 \leq g \leq 3$ and the embedding is by a non-special line bundle, $M_{d, g}(2)=\varnothing$, unless $(d, g)=(3,1)$.

Let $\mathcal{W}$ be the set of all smooth heptic hypersurfaces $W \subset \mathbb{P}^{5}$ satisfying the thesis of [7], i.e. containing no rational curve of degree $\leq 16$ (not even singular ones).

Let $Z \subset \mathbb{P}^{r}$ be any zero-dimensional scheme. For any hyperplane $H \subset \mathbb{P}^{r}$ let $\operatorname{Res}_{H}(Z)$ denote the residual scheme of $Z$ with respect of $H$, i.e. the closed subscheme of $\mathbb{P}^{r}$ with $\mathcal{I}_{Z}: \mathcal{I}_{H}$ as its ideal sheaf. We have $\operatorname{Res}_{H}(Z) \subseteq Z$ and $\operatorname{deg}(Z)=\operatorname{deg}\left(\operatorname{Res}_{H}(Z)\right)+\operatorname{deg}(H \cap Z)$. For every integer $t$ we have the following residual short exact sequence of coherent sheaves on $\mathbb{P}^{r}$ (the latter is also an $\mathcal{O}_{H}$-sheaf), which we often call the residual exact sequence of $H$ or of the inclusion $H \subset \mathbb{P}^{r}$ :

$$
0 \rightarrow \mathcal{I}_{\operatorname{Res}_{H}(Z)}(t-1) \rightarrow \mathcal{I}_{Z}(t) \rightarrow \mathcal{I}_{\mathrm{Z} \cap H, H}(t) \rightarrow 0
$$

Remark 1. Let $N \subset \mathbb{P}^{5}$ be a plane and $C \subset N$ be a smooth cubic. The normal bundle $N_{C}$ of $C$ in $\mathbb{P}^{5}$ is isomorphic to $\mathcal{O}_{C}(3) \oplus \mathcal{O}_{C}(1)^{\oplus 3}$ and hence $h^{1}\left(N_{C}\right)=0$ and $h^{0}\left(N_{C}\right)=18$. Hence the Hilbert scheme of $\mathbb{P}^{5}$ is smooth at $[C]$ and of dimension 18. We have $h^{1}\left(\mathcal{I}_{C}(t)\right)=0$ for all $t \in \mathbb{N}$ and hence $h^{0}\left(\mathcal{I}_{C}(7)\right)=\left(\begin{array}{c}12 \\ 5\end{array}\right)-21$. Hence a general heptic hypersurface contains no plane cubic.

By Remark 1 a general heptic hypersurface contains no element of $M_{d, g}(2)$. 
Remark 2. Let $N \subset \mathbb{P}^{5}$ be a plane and $C \subset N$ be a smooth degree 4 curve. $C$ has genus 3 and $\mathcal{O}_{C}(1) \cong \omega_{C}$. The normal bundle $N_{C}$ of $C$ in $\mathbb{P}^{5}$ is isomorphic to $\mathcal{O}_{C}(4) \oplus \mathcal{O}_{C}(1)^{\oplus 3}$ and hence $h^{1}\left(N_{C}\right)=3$ and $h^{0}\left(N_{C}\right)=23$. $\mathbb{P}^{5}$ has $\infty^{9}$ planes and each plane has $\infty^{14}$ degree 4 curves. Hence the Hilbert scheme of $\mathbb{P}^{5}$ is smooth at $[C]$ and of dimension 23. We have $h^{1}\left(\mathcal{I}_{C}(t)\right)=0$ for all $t \in \mathbb{N}$ and hence $h^{0}\left(\mathcal{I}_{C}(7)\right)=\left(\begin{array}{c}12 \\ 5\end{array}\right)-26$. Hence a general heptic hypersurface contains no degree 4 plane curves.

Lemma 1. Fix integer $t \geq 2, r \geq 3$ and an integral and non-degenerate curve $T \subset$ $\mathbb{P}^{r}$ such that $h^{1}\left(\mathcal{I}_{T}(t)\right)>0$. Fix a linear subspace $V \subseteq H^{0}\left(\mathcal{O}_{\mathbb{P}^{r}}(1)\right)$. Assume that $h^{1}\left(M, \mathcal{I}_{M \cap T, M}(t)\right)=0$ for every hyperplane $M \in|V|$. Then $h^{1}\left(\mathcal{I}_{T}(t-1)\right) \geq$ $h^{1}\left(\mathcal{I}_{T}(t)\right)+\operatorname{dim}(V)-1$.

Proof. For any hyperplane $M \subset \mathbb{P}^{r}$ we have an exact sequence

$$
0 \rightarrow \mathcal{I}_{T}(t-1) \rightarrow \mathcal{I}_{T}(t) \rightarrow \mathcal{I}_{T \cap M, M}(t) \rightarrow 0
$$

Since $h^{1}\left(M, \mathcal{I}_{T, M}(t)\right)=0$, the map $H^{1}\left(\mathcal{I}_{T}(t-1)\right) \rightarrow H^{1}\left(\mathcal{I}_{T}(t)\right)$ is surjective and hence its dual $e_{M}: H^{1}\left(\mathcal{I}_{T}(t)\right)^{\vee} \rightarrow H^{1}\left(\mathcal{I}_{T}(t-1)\right)^{\vee}$ is injective. Taking the equations of all hyperplanes we get a bilinear map map $u: H^{1}\left(\mathcal{I}_{T}(t)\right)^{\vee} \times$ $H^{0}\left(\mathcal{O}_{\mathbb{P}^{4}}(1)\right) \rightarrow H^{1}\left(\mathcal{I}_{T}(t-1)\right)^{\vee}$, which is injective with respect to the second variables, i.e. for every non-zero linear form $\ell u_{\mid H^{1}\left(\mathcal{I}_{T}(t)\right) \vee \times\{\ell\}}$ is injective (it is $e_{M}$ with $M:=\{\ell=0\})$. Hence if $(a, \ell) \in H^{1}\left(\mathcal{I}_{T}(t)\right)^{\vee} \times H^{0}\left(\mathcal{O}_{\mathbb{P}^{4}}(1)\right)$ with $a \neq 0$ and $\ell \neq 0$, then $u(a, \ell)=e_{M}(a) \neq 0$. Therefore the bilinear map $u$ is non-degenerate in each variable. Hence $h^{1}\left(\mathcal{I}_{T}(t-1)\right) \geq h^{1}\left(\mathcal{I}_{T}(t)\right)+\operatorname{dim}(V)-1$ by the bilinear lemma.

Lemma 2. Fix an integer $a>0$ and assume $d \geq 2 g-1+a$. Fix a zero-dimensional curvilinear scheme $Z \subset \mathbb{P}^{5}$ such that $\operatorname{deg}(Z)=$ a. Set $E_{Z}:=\left\{C \in M_{d, g}: Z \subset C\right\}$. Then every irreducible component of $E_{Z}$ has dimension $\leq 6 d+2-2 g-4 a$.

Proof. If $E_{Z}=\varnothing$, then the lemma is true. Hence we may assume $E \neq \varnothing$. Fix $C \in E$. By [27, Theoreme 1.5] it is sufficient to prove that $h^{1}\left(N_{C}(-Z)\right)=0$. Since $C$ is smooth, $N_{C}$ is a quotient of $T \mathbb{P}_{\mid C}^{5}$. By the Euler's sequence of $T \mathbb{P}^{5} N_{C}$ is a quotient of $\mathcal{O}_{C}(1)^{6}$. Since $d \geq 2 g-1+a$, we have $h^{1}\left(\mathcal{O}_{C}(1)(-Z)\right)=0$.

Lemma 3. Fix integers $t \geq 1$ and $r \geq 2$. Let $Z \subset \mathbb{P}^{r}$ denote a curvilinear zerodimensional scheme such that $c:=\operatorname{deg}(Z) \leq 3 t+r-2, Z$ spans $\mathbb{P}^{r}$ and $h^{1}\left(\mathcal{I}_{Z}(t)\right)>0$; if $c=3 t+r-2$ assume that $h^{1}\left(\bar{N}, \mathcal{I}_{N \cap Z, N}(t)\right)=0$ for every plane $N \subseteq \mathbb{P}^{r}$. Then either there is a line $L \subset \mathbb{P}^{r}$ with $\operatorname{deg}(L \cap Z) \geq t+2$ or there is a conic $D \subset \mathbb{P}^{r}$ with $\operatorname{deg}(D \cap Z) \geq 2 t+2$.

Proof. The case $r=2$ is true for all $t$ by [11, Corollaire 2] (in the case $c=3 t$ we assumed that both $h^{1}\left(\mathcal{I}_{Z}(t)\right)>0$ and $\left.h^{1}\left(\mathcal{I}_{Z}(t)\right)=0\right)$. Hence we may assume $r \geq 3$ and use induction on $r$. The case $t=1$ is true (if $c \leq r$, because no $Z$ with $\operatorname{deg}(Z) \leq r$ spans $\mathbb{P}^{r}$, while if $c=r+1$ because $h^{1}\left(\mathcal{I}_{Z}(x)\right)=0$ for all $x \geq 1$ if $\operatorname{deg}(Z)=r+1$ and $Z$ spans $\mathbb{P}^{r}$ ). Hence we may assume $t \geq 2$ and use induction on $t$ in $\mathbb{P}^{r}$.

(a) Let $M \subset \mathbb{P}^{r}$ be a hyperplane such that $a:=\operatorname{deg}(Z \cap M)$ is maximal. First assume $h^{1}\left(M, \mathcal{I}_{Z \cap M, M}(t)\right)>0$. Since $Z$ spans $M$ we have $\operatorname{deg}(Z \cap M) \leq$ 
$c-1$. The maximality property of $M$ gives that $Z \cap M$ spans $M$. Hence the inductive assumption gives that either there is a line $L \subset M$ with $\operatorname{deg}(L \cap Z) \geq t+2$ or there is a conic $D \subset M$ with $\operatorname{deg}(D \cap Z) \geq 2 t+2$. Hence we may assume $h^{1}\left(M, \mathcal{I}_{Z \cap M, M}(t)\right)=0$. The residual sequence (1) of $M$ gives $h^{1}\left(\mathcal{I}_{\operatorname{Res}_{M}(Z)}(t-1)\right)>0$. We have $\operatorname{deg}\left(\operatorname{Res}_{M}(Z)\right)=c-a$. Since $M \cap Z$ spans $M$ we have $a \geq r \geq 3$. Assume for the moment $a=r$. The maximality property of $M$ implies that $Z$ is in linearly general position in $\mathbb{P}^{r}$. Since $c \leq r t+1$, we get $h^{1}\left(\mathcal{I}_{Z}(t)\right)=0$ ([10, Theorem 3.2]). Hence $c-a \leq c-r-1 \leq 3 t+r-2-r-1 \leq$ $3(t-1)+r-3$. By the inductive assumption either there is a line $L \subset \mathbb{P}^{r}$ with $\operatorname{deg}(L \cap Z) \geq t+1$ or there is a conic $D \subset \mathbb{P}^{r}$ with $\operatorname{deg}(D \cap Z) \geq 2 t$. Assume the existence of a conic $D \subset \mathbb{P}^{r}$ with $\operatorname{deg}(D \cap Z) \geq 2 t$. Since $r-1 \geq 2$, the maximality property of $M$ gives $a \geq 2 t+r-3$. Hence $c \geq 2 t+2 t+r-3$, a contradiction. Now assume the existence of a line $L \subset \mathbb{P}^{r}$ with $\operatorname{deg}(L \cap Z) \geq t+1$. To prove the lemma we may assume $\operatorname{deg}(L \cap Z)=t+1$. Let $H \subset \mathbb{P}^{5}$ be a hyperplane containing $L$ and with $b:=\operatorname{deg}(H \cap Z)$ maximal among all hyperplanes containing $L$. Since $Z$ spans $\mathbb{P}^{r}$, we have $b \geq t+r-1$. If $h^{1}\left(H, \mathcal{I}_{Z \cap H, H}(t)\right)>0$, then we conclude by the inductive assumption on $r$. Hence we may assume $h^{1}\left(H, \mathcal{I}_{\mathrm{Z} \cap H, H}(t)\right)=0$. The residual sequence (1) of $H$ gives $h^{1}\left(\mathcal{I}_{\operatorname{Res}_{H}(Z)}(t-1)\right)>$ 0 . We have $\operatorname{deg}\left(\operatorname{Res}_{H}(Z)\right)=c-b \leq 2 t-1$. Hence the inductive assumption on $t$ gives the existence of a line $L \subset \mathbb{P}^{5}$ with $\operatorname{deg}\left(L \cap \operatorname{Res}_{H}(Z)\right) \geq t+1$. If the lemma fails, then $\operatorname{deg}\left(R \cap \operatorname{Res}_{H}(Z)\right)=\operatorname{deg}(R \cap Z)=t+1$. In this case we have $c \geq 2 t+2$. Hence the lemma is proved in degree $t$ if $c \leq 2 t+1$ (but this part is just [4, Lemma 34]).

(b) First assume $R \neq L$ and $R \cap L \neq \varnothing$. Since $\operatorname{deg}\left(R \cap \operatorname{Res}_{H}(Z)\right)=t+1$ and $H \supset L$, we have $\operatorname{deg}((L \cup R) \cap Z=2 t+2$. Hence we may take $D:=R \cup L$.

(c) Now assume $R=L$. We may take $Z$ minimal with the restriction that $h^{1}\left(\mathcal{I}_{Z}(t)\right)>0$ and $\operatorname{deg}(Z \cap L)=t+1$. Part (a) of our proof works if instead on $H$ we take any hyperplane $U \supset L$ (since as in the first part we exclude the existence of a conic $D$ with $\left.\operatorname{deg}\left(\operatorname{Res}_{U}(Z)\right) \geq 2 t\right)$. Let $Q$ be a quadric hypersurface containing $L$ in its singular locus. Since $\operatorname{deg}\left(\operatorname{Res}_{Q}(Z)\right) \leq 3 t-2 t-2 \leq t-2$, we have $h^{1}\left(\mathcal{I}_{\operatorname{Res}_{Q}(Z)}(t-2)\right)=0$ (even in the case $\left.t=2\right)$. Therefore the residual exact sequence of $Q$ gives $h^{1}\left(Q, \mathcal{I}_{Z \cap Q, Q}(t)\right)>0$ and hence $h^{1}\left(\mathcal{I}_{Z \cap Q}(t)\right)>0$. The minimality of $Z$ gives $Z \subset Q$. Since $Z$ is curvilinear, taking $Q=N_{1}+N_{2}$ with $N_{1}, N_{2}$ hyperplanes we also get that only the connected components of $Z$ whose reduction are contained in $L$ arise (for a minimal $Z$ ), hence we reduce to the case $\operatorname{deg}(Z)=2 t+2$. Let $W \subset Z$ be any degree $2 t+1$ subscheme. Since $\operatorname{deg}(W \cap J) \leq$ $\operatorname{deg}(Z \cap J) \leq t+1$ for each line $J$, part (a) of the proof gives $h^{1}\left(\mathcal{I}_{W}(t)\right)=0$. Hence $h^{1}\left(M, \mathcal{I}_{Z, M}(t)\right)=1$. Since $h^{1}\left(N, \mathcal{I}_{Z \cap N, N}(t)\right)=0$ for every hyperplane $N \subset \mathbb{P}^{r}$, Lemma 1 gives $h^{1}\left(\mathcal{I}_{Z}(t-1)\right) \geq r+h^{1}\left(\mathcal{I}_{Z}(t)\right)=r+1$. Let $N$ be any hyperplane plane containing $L$. We have $h^{1}\left(N, \mathcal{I}_{Z \cap N}(t-1)\right)=1$, because $\operatorname{deg}(Z \cap L)=t+1$ and $\operatorname{deg}(Z \cap N) \leq 2(t-1)+1$ (use the residual exact sequence (1) of a general hyperplane $M$ of $N$ containing $L$ in $N)$. Since $\operatorname{deg}\left(\operatorname{Res}_{N}(Z)\right) \leq t+1$, we have $h^{1}\left(\mathcal{I}_{\operatorname{Res}_{N}(Z)}(t-2)\right) \leq 2$ (part (a) applied. Hence the residual exact sequence (1) of $N$ gives $h^{1}\left(\mathcal{I}_{\operatorname{Res}_{N}(Z)}(t-1)\right) \leq 2+1$, a contradiction.

(d) Now assume $R \cap L=\varnothing$. First assume $r=3$. Let $Q \subset \mathbb{P}^{3}$ be a general quadric containg $L \cup R$. Since $\mathcal{I}_{L \cup R}(2)$ is spanned and $C$ is curvilinear, 
$Q \cap Z=Z \cap(R \cup L)$ (as schemes). Hence $h^{1}\left(Q, \mathcal{I}_{Z \cap Q, Q}(t)\right)=0$. The residual exact sequence of $Q$ gives $h^{1}\left(\mathcal{I}_{\operatorname{Res}_{Q}(Z)}(t-2)\right)>0$. Since $\operatorname{deg}\left(\operatorname{Res}_{Q}(t)\right) \leq t-2$, part (a) or [4, Lemma 34] gives a contradiction. Now assume $r \geq 4$. Since $L \cup R$ spans a 3-dimensional linear space, the maximality property of $M$ gives $b \geq 2 t+2+r-4$. Hence $c-b \leq t-1<\operatorname{deg}\left(R \cap \operatorname{Res}_{H}(Z)\right)$, a contradiction.

\section{C non-degenerate}

Le $\mathrm{t} M_{d, g}^{\prime}$ be the set of all $C \in M_{d, g}$ spanning $\mathbb{P}^{5} . M_{d, g}^{\prime}$ is smooth and irreducible and $\operatorname{dim}\left(M_{d, g}^{\prime}\right)=6 d+2-2 g$. A general non-special curve $C \subset \mathbb{P}^{5}$ of genus $g$ and degree $d \geq g+5$ has maximal rank ([3]). We have $d \leq 16$ and $7 \cdot 16+1-g<\left(\begin{array}{c}12 \\ 5\end{array}\right)$. Hence $h^{1}\left(\mathcal{I}_{C}(7)\right)=0$ for a general $C \in M_{d, g}$, i.e. $h^{0}\left(\mathcal{I}_{C}(7)\right)=\left(\begin{array}{c}12 \\ 5\end{array}\right)-7 d-1+g$. A dimensional count shows that no $C \in M_{d, g}^{\prime}$ is contained in a general heptic hypersurface. Hence in this section we only need to exclude all $C \in M_{d, g}^{\prime}$ with $h^{1}\left(\mathcal{I}_{C}(7)\right)>0$. By [13, Part (ii) of Theorem at page 492] we have $d \geq 13$.

Lemma 4. No $C \in M_{d, g^{\prime}}^{\prime} d \geq 11,1 \leq g \leq 3$, is contained in a degree 4 surface.

Proof. Fix a degree 4 surface $F \subset \mathbb{P}^{5}$ containing $C \in M_{d, g}^{\prime}$. Since $C$ is nondegenerate, $F$ is non-degenerate. By the classification of minimal degree surfaces either $F$ is a cone over a rational normal curve of $\mathbb{P}^{4}$ or it is an embedding of $F_{0}$ by the complete linear system $|h+2 f|$ or it is an embedding of $F_{2}$ by the complete linear system $|h+3 f|$.

(a) Assume that $F$ is an embedding of $F_{0}$ by the complete linear system $|h+2 f|$. Since $1 \leq g \leq 3$, either $C \in|2 h+(g+1) f|$ or $C \in|(g+1) h+2 f|$. In the first (second) case we get $d=5+g \leq 8$ (resp. $d=2 g+4 \leq 10$ ), a contradiction.

(b) Assume that $F$ is an embedding of $F_{2}$ by the complete linear system $|h+3 f|$ with $C \in|a h+b f|$. Since $g>0$, we have $a \geq 2$ and $b \geq 2 a$. We have $d=a+b$. Since $\omega_{F_{2}} \cong \mathcal{O}_{F_{2}}(-2 h-4 f)$, the adjunction formula gives $2 g-2=$ $a(-2(a-2)+(b-4))+b(a-2)$. If $a \geq 3$ and hence $b \geq 6$ we get $b \leq 2 g-2$, a contradiction. If $a=2$ and hence $b=d-2$, we get $2 g-2=2(d-6)$, a contradiction.

(c) Assume that $F$ is a cone over a rational normal curve of $\mathbb{P}^{4}$. Call $o$ its vertex and let $u: G \rightarrow F$ be the blowing up of $o$. $G \cong F_{4}$ and, up to this isomorphism, $u$ is induced by the complete linear system $|h+4 f|$. Let $C^{\prime} \subset F_{4}$ be the strict transform of $C$. Write $C^{\prime} \in|a h+b f|$. Since $C$ is smooth, $C^{\prime}$ is smooth and of genus $g>0$. Hence $a \geq 2$ and $b \geq 4 a$. We have $d=b$. Hence $2 \leq a \leq 4$ with $a=4$ only if $d=16$. Since $\omega_{F_{4}} \cong \mathcal{O}_{F_{4}}(-2 h-6 f)$, the adjunction formula gives $2 g-2=a(-4(a-2)+(b-6))+b(a-2) \geq b(a-2)$. Hence $a=2$ and $2 g-2=2(d-6)$, a contradiction.

Lemma 5. Fix $C \in M_{d, g}^{\prime}$ and let $H \subset \mathbb{P}^{5}$ be a general hyperplane.

(a) We have $h^{1}\left(H, \mathcal{I}_{\mathrm{C} \cap H, H}(3)\right) \leq \max \{0, d-13\}$.

(b) Assume $d \geq 14$. We have $h^{1}\left(H, \mathcal{I}_{C \cap H, H}(3)\right)=d-13$ if and only if $C \cap H$ is contained in a rational normal curve of $H$. The latter case does not occur for a curve $C$ contained in a general heptic hypersurface if $h^{1}\left(\mathcal{I}_{C}(7)\right) \leq 3 d-18-6 g$. 
Proof. The scheme $Z:=C \cap H$ is a set of $d$ points in uniform position and spanning $H$. Fix $S \subseteq Z$ with $\sharp(S)=\min \{d, 13\}$. Since $S$ is in linearly general position in $H=\mathbb{P}^{4}$, we have $h^{1}\left(H, \mathcal{I}_{S, H}(3)\right)=0$ ([10, Theorem 3.2]). Now assume $d \geq 14$. If $C \cap H$ is contained in a rational normal curve $D$ of $H$, then $h^{0}\left(H, \mathcal{I}_{Z, H}(3)\right) \leq h^{0}\left(H, \mathcal{I}_{D, H}(3)\right)=\left(\begin{array}{l}7 \\ 3\end{array}\right)-13$ and hence $h^{1}\left(H, \mathcal{I}_{Z, H}(3)\right) \geq d-13$. By part (a) the last inequality is an equality. Now assume $h^{1}\left(H, \mathcal{I}_{Z, H}(3)\right)=$ $d-13$. First assume $h^{0}\left(H, \mathcal{I}_{Z, H}(2)\right) \geq 6$. Since $Z$ is uniform position, we get $h^{0}\left(H, \mathcal{I}_{Z, H}(2)\right)=6$ and that $Z$ is contained in a rational normal curve of $H([15$, Lemma 3.9]). Now assume $h^{0}\left(H, \mathcal{I}_{Z, H}(2)\right) \leq 5$. Fix any $A \subset Z$ with $\sharp(A)=10$. Since $h^{0}\left(H, \mathcal{I}_{Z, H}(2)\right) \leq 5$, we have $h^{1}\left(H, \mathcal{I}_{A, H}(2)\right)=0$. Fix $B \subset Z \backslash A$ with $\sharp(B)=4$. Since $Z$ is in linearly general position, $B$ spans a hyperplane $N$ of $H$ and $Z \cap N=B$. We have $h^{1}\left(N, \mathcal{I}_{B, N}(3)\right)=0$. The exact sequence

$$
0 \rightarrow \mathcal{I}_{A, H}(2) \rightarrow \mathcal{I}_{A \cup B, H}(3) \rightarrow \mathcal{I}_{B, N}(3) \rightarrow 0
$$

gives $h^{1}\left(H, \mathcal{I}_{A \cup B, H}(3)\right)=0$ and hence $h^{1}\left(H, \mathcal{I}_{Z, H}(3)\right) \leq d-14$, a contradiction.

Now assume that $C \cap H$ is contained in a rational normal curve $T_{H}$ of $H$. Set $a:=d+1-2 g$. Fix $Z \subset T_{H}$ such that $\sharp(Z)=a$. By Lemma 2 the set of all $C \in M_{d, g}^{\prime}$ containing $Z$ has codimension at least $4 a$ in $M_{d, g}^{\prime}$. Since $\mathbb{P}^{5}$ has $\infty^{5}$ hyperplanes, each hyperplane has 15 rational normal curves and each rational normal curves has $\infty^{a}$ subsets with cardinality $a$, to rule out these cases it is sufficient to test all $C \in M_{d, g}^{\prime}$ with $h^{1}\left(\mathcal{I}_{C}(7)\right) \geq 3 a-20$.

Remark 3. Fix $C \in M_{d, g}^{\prime}$ and let $H \subset \mathbb{P}^{5}$ be a general hyperplane. The exact sequence (2) with $T:=C$ and $M:=H$ gives $h^{1}\left(\mathcal{I}_{C}(t-1)\right) \geq h^{1}\left(\mathcal{I}_{C}(t)\right)-$ $h^{1}\left(H, \mathcal{I}_{\mathrm{C} \cap H, H}(t)\right)$. Now assume $d \leq 4 t+1$. Since $C \cap H$ is in uniform position in $H$ and it spans $H$, it is in linearly general position. Hence $h^{1}\left(H, \mathcal{I}_{C \cap H, H}(t)\right)=0$ ([10, Theorem 3.2]).

Lemma 6. A general heptic hypersurface contains no $C \in M_{d, g^{\prime}}^{\prime} g>0$, with either $h^{0}\left(\mathcal{I}_{C}(2)\right) \geq 5, h^{0}\left(\mathcal{I}_{C}(3)\right) \neq 25$ and $d=15,16$, or $h^{0}\left(\mathcal{I}_{C}(2)\right) \geq 6$ and $13 \leq d \leq 16$.

Proof. Fix $C \in M_{d, g}^{\prime}$ with $h^{0}\left(\mathcal{I}_{C}(2)\right) \geq 5$. Let $K$ be the set-theoretic base locus of $\left|\mathcal{I}_{C}(2)\right|$. Since $C$ is irreducible, we have $\operatorname{dim}(K) \leq 3$. Fix an irreducible component $A$ of $K$ containing $C$ and with maximal dimension. Note that $h^{0}\left(\mathcal{I}_{A}(2)\right)=$ $h^{0}\left(\mathcal{I}_{C}(2)\right)$ and that $A=C$ if and only if $\operatorname{dim}(A)=1$.

(a) Assume $\operatorname{dim}(A) \geq 3$. Since $\operatorname{dim}(K) \leq 3$, we have $\operatorname{dim}(A)=3$ and $\operatorname{deg}(A) \geq 3$. Since $h^{0}\left(\mathcal{I}_{C}(2)\right)>2, A$ is not the complete intersection of 2 quadrics and hence $\operatorname{deg}(A)=3$. Since $A$ is a minimal degree 3 -fold, their classification (linearly normal rational scrolls and cones over a rational normal curve of $\mathbb{P}^{3}$ ), gives $h^{0}\left(\mathcal{I}_{A}(2)\right)=3$, a contradiction.

(b) Assume $\operatorname{dim}(A)=2$. Let $T$ be the intersection of 3 general elements of $\left|\mathcal{I}_{C}(2)\right|$. Since $A$ is non-degenerate, we have $4 \leq \operatorname{deg}(A) \leq 7$ (even if $T$ has a 3-dimensional component) by Bezout's theorem ([12, Theorem 2.2.5]). By Lemma 4 we may assume $\operatorname{deg}(A) \geq 5$. The exact sequence

$$
0 \rightarrow \mathcal{I}_{A}(1) \rightarrow \mathcal{I}_{A}(2) \rightarrow \mathcal{I}_{A \cap H, H}(2) \rightarrow 0
$$


gives $h^{0}\left(\mathcal{I}_{C}(2)\right)=h^{0}\left(\mathcal{I}_{A}(2)\right) \leq h^{0}\left(H, \mathcal{I}_{A \cap H, H}(2)\right)$. The integral curve $A \cap H$ spans $H$. Set $q:=p_{a}(A \cap H)$. We have $q \leq \operatorname{deg}(A \cap C)-4$ for all $\operatorname{deg}(A) \in$ $\{5,6,7\}$ by an elementary case of the Castelnuovo' $s$ bound for the arithmetic genus of a curve in $\mathbb{P}^{r}$. By [13, part (ii) of Theorem at page 492] if $\operatorname{deg}(A) \leq 6$, then $h^{1}\left(H, \mathcal{I}_{A \cap H, H}(2)\right)=0$, except the case $\operatorname{deg}(A)=6, A \cap H$ a smooth rational curve having a quadrisecant line. Assume for the moment $\operatorname{deg}(A) \leq 6$ and that we are not in this exceptional case. We get $h^{0}\left(\mathcal{I}_{A \cap H, H}(2)\right)=14-2 \operatorname{deg}(A)+q \leq$ 4 , unless $\operatorname{deg}(A)=5$ and $q=1$.

(b1) Assume $\operatorname{deg}(A)=5$ and $q=1$. We may assume $h^{0}\left(\mathcal{I}_{A}(2)\right)=5$. So we do need to check this case if $d=13,14$ and hence here we assume $d \in\{15,16\}$. In this case $A \cap H$ is linearly normal and arithmetically Cohen-Macaulay with $h^{0}\left(H, \mathcal{I}_{A \cap H, H}(3)\right)=35-15=20$. Since $h^{0}\left(\mathcal{I}_{A}(2)\right)=h^{0}\left(H, \mathcal{I}_{A \cap H, H}(2)\right)$, then $A$ is linearly normal by (3). By (3) we get that $A$ is arithmetically Cohen-Macaulay and in particular $h^{1}\left(\mathcal{I}_{A}(2)\right)=0$. From (3) we get $h^{0}\left(\mathcal{I}_{A}(3)\right)=h^{0}\left(\mathcal{I}_{A}(2)\right)+$ $h^{0}\left(H, \mathcal{I}_{A \cap H, H}(3)\right)=25$. Since $C \subset A$, we get $h^{0}\left(\mathcal{I}_{C}(3)\right) \geq 25$. By assumption $h^{0}\left(\mathcal{I}_{C}(2)\right) \neq 25$ and so $h^{0}\left(\mathcal{I}_{C}(3)\right) \geq 26$. Therefore there is a cubic hypersurface $T \subset \mathbb{P}^{5}$ with $C \subset T$ and $A \nsubseteq T$, so that $C$ is contained in the locally CohenMacaulay curve $A \cap T$. Since $\operatorname{deg}(A \cap T)=15$, we get $d \neq 16$ and $C=A \cap T$. Since $\omega_{A \cap T} \cong \mathcal{O}_{A \cap T}(2)$, we get $g>3$, a contradiction.

(b2) Now assume $\operatorname{deg}(A)=6$ and that $D:=A \cap H$ is a smooth rational curve spanning $H$ and with a line $L \subset H$ with $\operatorname{deg}(L \cap D)=4$. We have $L \subset K, p_{a}(L \cup D)=3$ and $L \cup D$ is a linearly normal curve of $H=\mathbb{P}^{4}$. Let $N \subset H$ be a general hyperplane. Since $h^{i}\left(H, \mathcal{I}_{D \cup L}(1)\right)=0, i=0,1$, a standard exact sequence gives $h^{0}\left(H, \mathcal{I}_{D \cup L}(2)\right)=h^{0}\left(N, \mathcal{I}_{N \cap(D \cup L), N}(1)\right)$ and hence $h^{0}\left(H, \mathcal{I}_{D, H}(2)\right)=h^{0}\left(H, \mathcal{I}_{D \cup L, H}(2)\right) \leq h^{0}\left(N, \mathcal{I}_{N \cap D, N}(2)\right)$. Since $N \cap D$ is in uniform position in $N$ and it spans $N$, we get $h^{1}\left(N, \mathcal{I}_{N \cap D, N}(2)\right)=0$ and hence $h^{0}\left(N, \mathcal{I}_{D \cap N, N}(2)\right)=4$. Hence $h^{0}\left(\mathcal{I}_{C}(2)\right) \leq 4$, a contradiction.

(b3) Now assume $\operatorname{deg}(A)=7$. By Bezout's theorem ([12, Theorem 2.2.5]) we have $T \cap H=(A \cap H) \cup L_{H}$ with $L_{H}$ a linear space of dimension $\geq 1$. If $L_{H}$ is a line, then the complete intersection $T \cap H$ links $A \cap H$ to a line and hence $A \cap H$ is arithmetically Cohen-Macaulay. We get $h^{0}\left(\mathcal{I}_{C}(2)\right)=h^{0}\left(\mathcal{I}_{C \cap H}(2)\right)=1+q \leq 4$. Now assume $\operatorname{dim}\left(L_{H}\right)>1$. Since $T$ is a general intersection of 3 elements of $\left|\mathcal{I}_{C}(2)\right|$ and $H$ is general, we first get that $K$ has a 3-dimensional component, $B$, which is a linear space, then we get $T=A \cup B$ and then we get $A \cup B=K$. Hence $h^{0}\left(\mathcal{I}_{C}(2)\right)=3$, a contradiction.

(c) Assume $\operatorname{dim}(A)=1$, i.e. $A=C$, and $d=15,16$. Let $T$ be the intersection of 3 general elements of $\left|\mathcal{I}_{C}(2)\right|$. Let $B$ be an irreducible component of $T_{\text {red }}$ containing $C$. Since we may apply steps (a) and (b) to every irreducible component of $K$ containing $C$, we have $B \nsubseteq K$ and hence there is a quadric containing $C$, but not containing $B$. By Bezout we have $\operatorname{deg}(B) \leq 8$ with equality if and only if $\operatorname{dim}(T)=2$ and $B=T$. Intersecting $B$ with a general element of $\left|\mathcal{I}_{C}(2)\right|$ we get a locally Cohen-Macaulay scheme $E$ of pure dimension 1 with $\operatorname{deg}(E) \leq 16$ and $E \supseteq C$. We exclude the case $d=16$, because $C$ has not the genus, 17, of an intersection of 4 quadrics. Now assume $d=15$. Since $B=T, T$ has dimension 2 and hence it is a complete intersection. Hence the complete intersection $E$ links $C$ to a line. Therefore $C$ is arithmetically Cohen-Macaulay and in particular $h^{1}\left(\mathcal{I}_{C}(2)\right)=0$, contradicting the inequality $2 d+1-g>21$. 
(d) Assume $d=13,14$. Take $K, A$ as in the previous steps. Fix a general hyperplane $H \subset \mathbb{P}^{5}$. Since $C$ is non-degenerate, we have $h^{0}\left(\mathcal{I}_{C}(1)\right)=0$. By (3) with $C$ instead of $A$ we have $h^{0}\left(\mathcal{I}_{C}(2)\right) \leq h^{0}\left(H, \mathcal{I}_{C \cap H, H}(2)\right)$. Assume $h^{0}\left(H, \mathcal{I}_{C \cap H, H}(2)\right) \geq 6$. By [15, Lemma 3.9] $C \cap H$ is contained in a rational normal curve $D \subset H$. Since $d>8$, we have $D \subset K$. By step (a) we get the existence of $A \subseteq K$ with $C \subset A$ and $A$ a degree 4 surface, contradicting Lemma 4 .

Lemma 7. Fix integers $d, g$ such that $6 \leq g+5 \leq d \leq 15$. Let $\Gamma$ be the set of all $C \in M_{d, g}^{\prime}$ contained in the smooth locus a quadric. For any $v \in\{0,1,2\}$ and integer $x>0$ let $\Gamma_{v, x}$ be the set of all $C \in M_{d, g}^{\prime}$ contained in a quadric with singular locus $V$ of dimension $v$ and $x=\operatorname{deg}(V \cap C)$. Then $\operatorname{dim}(\Gamma) \leq 4 d+21+g, \Gamma_{0, x}=\varnothing$ for all $x \geq 2, \operatorname{dim}\left(\Gamma_{0,1}\right) \leq 4 d+22+g, \operatorname{dim}\left(\Gamma_{1, x}\right) \leq 4 d+g+x+18$, and $\operatorname{dim}\left(\Gamma_{2, x}\right) \leq$ $4 d+14+g$.

Proof. Fix $C \in M_{d, g}^{\prime}$ with $h^{0}\left(\mathcal{I}_{C}(2)\right)>0$ and let $Q \subset \mathbb{P}^{5}$ be any quadric containing C. Since $C$ is non-degenerate, $Q$ is irreducible. The Hilbert scheme $\operatorname{Hilb}(Q)$ of $Q$ has $H^{0}\left(N_{C, Q}\right)$ as its tangent space at $[C]$. Let $V$ be the singular locus of $Q$. Let $\tau$ be the tangent sheaf of $Q$. Since the algebraic group $\operatorname{Aut}(Q)$ acts transitively on $Q \backslash V$ and $H^{0}(\tau)$ is the tangent space to $\operatorname{Aut}(Q)$ at the identity, $H^{0}(\tau)$ spans $\tau$ at each point of $Q \backslash V$.

(i) First assume that either $Q$ is smooth or $C \cap V=\varnothing$. Since $\operatorname{dim}\left|\mathcal{O}_{\mathbb{P}^{5}}(2)\right|=$ 20 , to handle these curves $C$ it is sufficient to prove that the set of all $C \in M_{d, g}$ contained in $Q$ and with $V \cap Q=\varnothing$ has dimension $\leq 4 d+1+g$. Since $C \cap V=$ $\varnothing$, the normal sheaf $N_{C, Q}$ is a rank 3 vector bundle on $C$ with degree $4 d+2 g-2$. Since $N_{C, Q}$ is a quotient of $\tau_{\mid C}$, it is spanned. Take 2 general sections of $H^{0}\left(N_{C, Q}\right)$. These sections induces a rank 2 subsheaf $\mathcal{G}^{\prime}$ of $N_{C, Q}$ isomorphic to $\mathcal{O}_{C}^{\oplus 2}$. Let $\mathcal{G}$ be the saturation of $\mathcal{G}^{\prime}$ in $N_{C, Q}$, i.e. the only rank 2 subsheaf of $N_{C, Q}$ containing $\mathcal{G}^{\prime}$ and with $N_{C, Q} / \mathcal{G}$ a line bundle. Since $h^{1}\left(\mathcal{G}^{\prime}\right)=2 g$, we have $h^{1}(\mathcal{G}) \leq 2 g$. First assume $\operatorname{deg}(\mathcal{G}) \leq 3 d-1$, i.e. $\operatorname{deg}\left(N_{C, Q} / \mathcal{G}\right)>2 g-2$. We get $h^{1}\left(N_{C, Q} / \mathcal{G}\right)=$ 0 and so $h^{1}\left(N_{C, Q}\right) \leq 2 g$. Riemann Roch gives $h^{0}\left(N_{C, Q}\right) \leq 4 d+g+1$. Now assume $\operatorname{deg}(\mathcal{G}) \geq 3 d$. Let $\mathcal{G}_{1}$ be the saturation of a general section of $\mathcal{G}$. So $\mathcal{G}$ is the extension of two line bundles, $\mathcal{G} / \mathcal{G}_{1}$ and $\mathcal{G}_{1}$, with $\operatorname{deg}\left(\mathcal{G} / \mathcal{G}_{1}\right)+\operatorname{deg}\left(\mathcal{G}_{1}\right)=$ $3 d \geq 2(2 g-1)$ and both with a non-zero section. We get that at least one of the line bundles $\mathcal{G} / \mathcal{G}_{1}$ and $\mathcal{G}_{1}$ is non-special and the other one, $\mathcal{L}$, has $h^{1}(\mathcal{L}) \leq g$. Hence $h^{1}(\mathcal{G}) \leq g$. Since the line bundle $N_{C, Q} / \mathcal{G}$ has a non-zero section, we have $h^{1}\left(N_{C, Q} / \mathcal{G}\right) \leq g$ and hence $h^{1}\left(N_{C, Q}\right) \leq 2 g$ even in this case.

(ii) Now assume that $C \cap V \neq \varnothing$. The set of all quadrics of rank 5 (resp. 4, resp. 3) has dimension 19 (resp. 17, resp. 14). Since $C$ is not a plane curve and $\operatorname{dim}(V) \leq 2$, the scheme $C \cap V$ is finite. Let $u: \widetilde{Q} \rightarrow Q$ be the blowing up of $V, E:=u^{-1}(V)$ the exceptional divisor, and $\widetilde{C} \subset \widetilde{Q}$ the strict transform of $C$. Since $C$ is smooth, $u$ maps isomorphically $\widetilde{C}$ onto $C$. Let $\Psi_{v, x}$ be closure in $\operatorname{Hilb}(\widetilde{Q})$ of the strict transforms of all $C \subset Q$ with $\operatorname{deg}(C \cap V)=x$. Take a general $D \in \Psi_{v, x}$. Since $\operatorname{Aut}(\widetilde{Q})$ acts transitively of $\widetilde{Q} \backslash E$, step (a) of the proof gives $h^{1}\left(N_{D, \widetilde{Q}}\right) \leq 2 g$. Hence it is sufficient to give a winning upper bound for $\operatorname{deg}\left(N_{D, \widetilde{Q}}\right)$, i.e. a winning lower bound for $\operatorname{deg}\left(\omega_{\widetilde{Q} \mid \widetilde{C}}\right)$. The group $\operatorname{Pic}(\widetilde{Q})$ is freely generated by $E$ and the pull-back $H$ of $\mathcal{O}_{Q}(1)$. We have $D \cdot H=d$ and $D \cdot E=x$. 
We claim that $\omega_{\widetilde{Q}} \cong \mathcal{O}_{\widetilde{Q}}(-3 H+c E)$ with $c=-2+v$. First assume $v>0$. Let $M \subset Q$ be a general hyperplane. $M \cap Q$ is a 3-dimensional quadric with vertex of dimension $v-1$. We apply [18], Example 8.5 (2), if $v=1$ and [18], Example 8.5 (3) if $v=2$. Now assume $v=0$. To apply the previous formulas we need to take a hyperplane $M \supset V$ whose pull-back has $E$ as a component.

Lemma 8. A general heptic hypersurface contains no $C \in M_{d, g^{\prime}}^{\prime} 13 \leq d \leq 16$, $1 \leq g \leq 3$, with no line $R \subset \mathbb{P}^{5}$ with $\operatorname{deg}(R \cap C) \geq 7$ and no conic $D \subset \mathbb{P}^{5}$ with $\operatorname{deg}(C \cap D) \geq 12$.

Proof. Recall that $h^{0}\left(\mathcal{O}_{\mathbb{P}^{5}}(2)\right)=21$ and that we are assuming $h^{1}\left(\mathcal{I}_{C}(7)\right)>0$. By the cases $r=4, c \leq 3 t+1$ and $t=5,6,7$ of Lemma 3 we have $h^{1}\left(M, \mathcal{I}_{C \cap M, M}(t)\right)=$ 0 for all hyperplanes $M \subset \mathbb{P}^{5}$. Lemma 1 gives $h^{1}\left(\mathcal{I}_{C}(4)\right) \geq h^{1}\left(\mathcal{I}_{C}(5)\right)+5 \geq$ $h^{1}\left(\mathcal{I}_{C}(6)\right)+10 \geq h^{1}\left(\mathcal{I}_{C}(7)\right)+15$. Fix a general hyperplane $H \subset \mathbb{P}^{5}$. Lemma 5 and Remark 3 give $h^{1}\left(\mathcal{I}_{C}(3)\right) \geq h^{1}\left(\mathcal{I}_{C}(4)\right)$ and that either $h^{1}\left(\mathcal{I}_{C}(2)\right) \geq$ $h^{1}\left(\mathcal{I}_{C}(3)\right)-\max \{0, d-14\}$ or $d \geq 14, h^{1}\left(\mathcal{I}_{C}(2)\right) \geq h^{1}\left(\mathcal{I}_{C}(3)\right)+13-d$ and $h^{1}\left(\mathcal{I}_{C}(7)\right) \geq 3 d-18-g$. First assume $d \geq 14, h^{1}\left(\mathcal{I}_{C}(2)\right) \geq h^{1}\left(\mathcal{I}_{C}(3)\right)+13-d$ and $h^{1}\left(\mathcal{I}_{C}(7)\right) \geq 3 d-18-g$. We first get $h^{1}\left(\mathcal{I}_{C}(3)\right) \geq h^{1}\left(\mathcal{I}_{C}(4)\right) \geq 3 d-3-g$ and then $h^{1}\left(\mathcal{I}_{C}(2)\right) \geq 2 d+10-g$, i.e. $h^{0}\left(\mathcal{I}_{C}(2)\right) \geq 31$, contradicting Lemma 6 .

Now assume $h^{1}\left(\overline{\mathcal{I}}_{C}(2)\right) \geq h^{1}\left(\mathcal{I}_{C}(3)\right)-\max \{0, d-14\}$ and hence $h^{1}\left(\mathcal{I}_{C}(2)\right) \geq$ $16-\max \{0, d-14\}$, i.e. $h^{0}\left(\mathcal{I}_{C}(2)\right) \geq 36+g-2 d-\max \{0, d-14\}$. We get $h^{0}\left(\mathcal{I}_{C}(2)\right) \geq 21+16-1-26+g=11+g \geq 7$ if $d=13$ and $h^{0}\left(\mathcal{I}_{C}(2)\right) \geq$ $21+16-d+14-2 d-1+g=50-3 d+g$ if $14 \leq d \leq 16$. If either $13 \leq d \leq 15$ or $d=16$ and $g=3$ we use Lemma 6, except that if $d=16$ and $g=3$ we also need to exclude that $h^{0}\left(\mathcal{I}_{C}(3)\right)=25$. Assume $d=16$ and $g=3$. Since $h^{1}\left(\mathcal{I}_{C}(3)\right) \geq 16$ and $\left(\begin{array}{l}8 \\ 3\end{array}\right)=56$, we have $h^{0}\left(\mathcal{I}_{C}(3)\right) \geq 56+16-1-48+3=26$.

Now assume $d=16$ and $g=1,2$. We proved that $h^{0}\left(\mathcal{I}_{C}(2)\right) \geq 2+g$. To repeat the same proof it would be sufficient to have $h^{1}\left(\mathcal{I}_{C}(7)\right) \geq 4$. If $C$ is contained in the smooth locus of at least one quadric, then by Lemma 7 we may assume $h^{1}\left(\mathcal{I}_{C}(7)\right) \geq 6 d+2-2 g-(4 d-21+g)=2 d-19-3 g \geq 17$. Now assume that a general quadric hypersurface containing $C$ has singular locus $V$ of dimension $v$ and with $x:=\operatorname{deg}(C \cap V)>0$. Since $x \leq 6$ if $v=1$, it is sufficient to use Lemma 7 .

Notation 1. For each integer $a>0$ let $\mathcal{A}_{a}^{\prime}$ denote the set of all $C \in M_{d, g}^{\prime}$ such that there is a line $L \subset \mathbb{P}^{5}$ with $\operatorname{deg}(L \cap C)=a$ and $\mathcal{A}_{a}^{\prime \prime}:=\cup_{b \geq a} \mathcal{A}_{a}^{\prime}$. For each integer $a>0$ let $\mathcal{B}_{a}^{\prime}$ denote the set of all $C \in M_{d, g}^{\prime}$ such that there is a conic $D \subset \mathbb{P}^{5}$ with $\operatorname{deg}(L \cap D)=a$ and $\mathcal{B}_{a}^{\prime \prime}:=\cup_{b \geq a} \mathcal{B}_{a}^{\prime}$.

Lemma 9. A general $W \in \mathcal{W}$ contains no $C \in M_{d, g^{\prime}}^{\prime} 13 \leq d \leq 16,1 \leq g \leq 3$, with $\mathcal{A}_{7}^{\prime \prime} \neq \varnothing$.

Proof. (a) In this step we prove that $W$ contains no element of $\mathcal{A}_{9}^{\prime \prime}$. Fix $C \in \mathcal{A}_{9}^{\prime \prime}$ and take a line $L \subset \mathbb{P}^{5}$ such that $\operatorname{deg}(L \cap C) \geq 9$. Set $b:=8$ if $(d, g)=(13,3)$ and $b:=9$ if $(d, g) \neq(13,3)$. Fix $Z \subseteq C \cap L$ such that $\operatorname{deg}(Z)=9$. Set $E_{Z}:=$ $\left\{A \in M_{d, g}: A \supset Z\right\}$. Lemma 2 gives that $\operatorname{dim}\left(E_{Z}\right) \leq 6 d+2-2 g-4 b$. Since $L$ has $\infty^{b}$ degree $b$ subschemes and $\mathbb{P}^{5}$ has $\infty^{8}$ lines, to prove that no element of $\mathcal{A}_{9}^{\prime \prime}$ is contained in $W$ it is sufficient to test the ones, $C$, with the additional 
condition that either $h^{1}\left(\mathcal{I}_{C}(7)\right) \geq 19($ case $(d, g) \neq(13,3))$ or $h^{1}\left(\mathcal{I}_{C}(7)\right) \geq 16$ (case $(d, g)=(13,3))$. By the cases $t=3,4,5,6,7$ of Remark 3 we get $h^{1}\left(\mathcal{I}_{C}(3)\right) \geq$ $h^{1}\left(\mathcal{I}_{C}(7)\right)$. Therefore $h^{1}\left(\mathcal{I}_{C}(2)\right) \geq 17$ if $(d, g) \neq(13,3)$ and $h^{1}\left(\mathcal{I}_{C}(2)\right) \geq 16$ if $(d, g)=(13,3)$ (Lemma 5). We get $h^{0}\left(\mathcal{I}_{C}(2)\right) \geq 37+g-2 d \geq 6$ if $(d, g) \neq(13,3)$ and $h^{0}\left(\mathcal{I}_{C}(3)\right) \geq 13$ if $(d, g)=(13,3)$. Apply Lemma 6 .

(b) In this step we prove that a general heptic contains no element of $\mathcal{A}_{8}^{\prime}$. By part (a) it is sufficient to exclude all elements of $\mathcal{A}_{8}^{\prime} \backslash \mathcal{A}_{9}^{\prime \prime}$. By Lemma 2 it is sufficient to exclude all $C \in \mathcal{A}_{8}^{\prime} \backslash \mathcal{A}_{9}^{\prime \prime}$ with $h^{1}\left(\mathcal{I}_{C}(7)\right) \geq 16$. Fix any such $C$. By Lemma 1 and the case $r=4, t=7$ and $c=d \leq 3 t+1$ of Lemma 3 we have $h^{1}\left(\mathcal{I}_{C}(6)\right) \geq 21$. By the cases $t=3,4,5,6$ of Remark 3 we get $h^{1}\left(\mathcal{I}_{C}(3)\right) \geq 21$. Lemma 3 gives $h^{1}\left(\mathcal{I}_{C}(2)\right) \geq 19$ and hence $h^{0}\left(\mathcal{I}_{C}(2)\right) \geq 39-2 d+g \geq 8$. Use Lemma 6.

(c) Fix $C \in \mathcal{A}_{7}^{\prime} \backslash \mathcal{A}_{9}^{\prime \prime}$. By Lemma 2 it is sufficient to exclude the curves $C$ with $h^{1}\left(\mathcal{I}_{C}(7)\right) \geq 13$. Since $C \notin \mathcal{A}_{9}^{\prime \prime}$, we have $h^{1}\left(\mathcal{I}_{C}(6)\right) \geq 18$ (Lemma 1$)$. The case $t=4,5,6$ of Remark 3 gives $h^{1}\left(\mathcal{I}_{C}(3)\right) \geq 18$. Lemma 5 gives $h^{1}\left(\mathcal{I}_{C}(2)\right) \geq 16$ and hence $h^{0}\left(\mathcal{I}_{C}(2)\right) \geq 36+g-2 d$. Apply Lemma 6, except that if $d=16$ and $g=1$ we also need to prove that $h^{0}\left(\mathcal{I}_{C}(3)\right) \neq 25$. Since $h^{1}\left(\mathcal{I}_{C}(3)\right) \geq 18$, we have $h^{0}\left(\mathcal{I}_{C}(3)\right) \geq 56+18-48=26$.

Lemma 10. A general $W \in \mathcal{W}$ contains no $C \in M_{d, g}^{\prime}$ such that either there is a conic $D \subset \mathbb{P}^{5}$ with $\operatorname{deg}(D \cap C) \geq 12$ or there is a line $L \subset \mathbb{P}^{5}$ with $\operatorname{deg}(L \cap C) \geq 6$.

Proof. By Lemma 9 it is sufficient to exclude all $C \in \mathcal{B}_{12}^{\prime} \backslash \mathcal{A}_{7}^{\prime \prime}$ and all $C \in \mathcal{A}_{6}^{\prime} \backslash \mathcal{A}_{7}^{\prime \prime}$. (a) We first exclude all $C \in \mathcal{B}_{14}^{\prime \prime} \backslash \mathcal{A}_{7}$. In this case we have $d=16$ and $C \in \mathcal{B}_{14}^{\prime}$. Take a conic $D \subset \mathbb{P}^{5}$ such that $\operatorname{deg}(D \cap C)=14$. Since $C \notin \mathcal{A}_{7}^{\prime \prime}, D$ is a smooth conic. Set $x(1):=14, x(2):=13$ and $x(3):=11$. Fix any $Z \subset D$ such that $\operatorname{deg}(Z)=x(g)$. Since $x(g)+2 g-1 \leq 16$, the set $E_{Z}$ of all non-degenerate $A \in M_{16, g}$ with $Z \subset A$ has dimension $6 d+2-2 g-4 x(g)$ (Lemma 2). Since $D$ is a smooth curve, it has $\infty^{x(g)}$ degree $x(g)$ subschemes. $\mathbb{P}^{5}$ has $\infty^{9}$ planes and each plane has $\infty^{5}$ conics. Hence it is sufficient to exclude all non-degenerate $C \in \mathcal{B}_{14}^{\prime} \backslash \mathcal{A}_{7}$ with $h^{1}\left(\mathcal{I}_{C}(7)\right) \geq 3 x(g)-14$. By Lemma 1 and the case $r=4, t=7$, $c=d \leq 3 t+1$ of Lemma 3 (note that $\operatorname{deg}\left(C \cap D^{\prime}\right)<16$ for each conic $D^{\prime}$ and that $\left.\mathcal{A}_{9}^{\prime \prime}=\varnothing\right)$ we have $h^{1}\left(\mathcal{I}_{C}(6)\right) \geq 3 x(g)-9 \geq 22$. Then we continue as in the last two lines of step (a) of the proof of Lemma 9.

(b) Now we exclude all $C \in \mathcal{A}_{6}^{\prime} \backslash \mathcal{A}_{7}^{\prime \prime}$. Since $13 \geq 6+2 g-1$, by Lemma 2 we may assume $h^{1}\left(\mathcal{I}_{C}(7)\right) \geq 10$. By step (a) we may assume that $C \notin \mathcal{B}_{12}^{\prime \prime}$. Lemma 1 and the cases $t=6,7, r=4$, of Lemma 3 give $h^{1}\left(\mathcal{I}_{C}(5)\right) \geq 5+h^{1}\left(\mathcal{I}_{C}(6)\right) \geq$ $10+h^{1}\left(\mathcal{I}_{C}(7)\right) \geq 20$. Then we continue as in the proof of Lemma 9 .

(c) Now we exclude all $C \in \mathcal{B}_{12}^{\prime} \cup \mathcal{B}_{13}^{\prime} \backslash \mathcal{A}_{7}$. By step (b) we may assume $C \notin \mathcal{A}_{6}^{\prime}$. Take a conic $D$ such that $\operatorname{deg}(D \cap C) \in\{12,13\}$. Note that if $\mathcal{B}_{12}^{\prime \prime} \neq \varnothing$, then $d \neq 13$. Since $C \notin \mathcal{A}_{6}^{\prime \prime}$, the conic $D$ is smooth. Set $y_{d, g}:=\min \{12, d+1-2 g\}$. Fix any zero-dimensional scheme $Z \subset D$ with $\operatorname{deg}(Z)=y_{d, g}$. By Lemma 2 the set of all non-degenerate $C$ containing $Z$ has dimension $\leq 6 d+2-2 g-4 y_{d, g}$. Every smooth conic has $\infty^{y_{d, g}}$ zero-dimensional schemes of degree $y_{d, g} \cdot \mathbb{P}^{5}$ has $\infty^{9}$ planes and each plane has $\infty^{5}$ conics. Hence it is sufficient to check the curves $C$ with the additional condition $h^{1}\left(\mathcal{I}_{C}(7)\right) \geq 3 y_{d, g}-14$. Since $13 \leq d \leq 16$ and $g \leq 3$, we have $y_{d, g} \geq 8$ and so $3 y_{d, g}-14 \geq 10$. We conclude as in step (b). 
By Lemmas 8 and 10 we proved the part of Theorem 1 concerning non-degenerate $C \in M_{d, g}$.

\section{Curves spanning a hyperplane}

In this section we consider curves $C \in M_{d, g}$ spanning a hyperplane. For any hyperplane $M \subset \mathbb{P}^{5}$ let $M_{d, g}^{\prime}(M)$ be the set of all curves $C \in M_{d, g}$ spanning $M$. $M_{d, g}^{\prime}(M)$ is a smooth and irreducible variety of dimension $5 d+1-g$. A general element of $M_{d, g}^{\prime}(M)$ has maximal rank ([1]). Since $\left(\begin{array}{c}11 \\ 4\end{array}\right)>7 d+1-g$, we have $h^{1}\left(M, \mathcal{I}_{C, M}(7)\right)=0$ for a general $C \in M_{d, g}^{\prime}(M)$. Since $\mathbb{P}^{5}$ has $\infty^{5}$ hyperplanes to prove that a general $W \in \mathcal{W}$ contains no curve in $M_{d, g}$ spanning a hyperplane it is sufficient to fix a hyperplane $M \subset \mathbb{P}^{5}$ and exclude all $C \in M_{d, g}^{\prime}(M)$ such that $h^{1}\left(M, \mathcal{I}_{C, M}(7)\right) \geq d-4-g$.

Since $g>0$, by [13, part (ii) of Theorem on page 492] we have $h^{1}\left(M, \mathcal{I}_{C, M}(7)\right)=$ 0 if $d \leq 11$. Hence in this section we assume $12 \leq d \leq 16$ and we fix the hyperplane $M \subset \mathbb{P}^{5}$.

Remark 4. For any $C \in M_{d, g}^{\prime}(M)$ let $\alpha(C)$ (or just $\alpha$ ) denote the minimal integer $t$ such that $h^{0}\left(M, \mathcal{I}_{C, M}(t)\right)>0$. Since $C$ spans $M$, we have $\alpha \geq 2$. Since $d \leq 16$, we have $4 d+1-g<70=\left(\begin{array}{l}8 \\ 4\end{array}\right)$ and so $\alpha \leq 4$. Since $\left(\begin{array}{l}6 \\ 2\end{array}\right)=15$ and $\left(\begin{array}{l}7 \\ 3\end{array}\right)=35$, we have $\alpha=2$ if and only if $h^{1}\left(M, \mathcal{I}_{C, M}(2)\right) \geq 2 d-g-13$ and $\alpha \leq 3$ if and only if $h^{1}\left(M, \mathcal{I}_{C, M}(3)\right) \geq 3 d-g-33$.

Lemma 11. Fix $C \in M_{d, g}^{\prime}(M), d \leq 16, g>0$. There is no plane $N \subset M$ with $\operatorname{deg}(N \cap C) \geq 15$.

Proof. Assume the existence of a plane $N \subset M$ such that $\operatorname{deg}(N \cap C) \geq 15$. Fix a hyperplane $H \subset M$ with $H \supset N$. Since the scheme $C \cap H$ spans $H$, we get $d=16$ and $\operatorname{deg}(C \cap N)=15$. Since $C$ is smooth, for a general $H \supset N, H$ contains a tangent line of $C$ if and only if this tangent line is contained in $N$. Hence for a general $H$ we have $C \cap H=(C \cap N) \cup\left\{p_{H}\right\}$ with $p_{H} \in C \backslash C \cap N$. The pencil of all hyperplanes $H \supset N$ shows that $C$ is rational, a contradiction.

Lemma 12. A general $W \in \mathcal{W}$ contains no $C \in M_{d, g}$ such that $C \in M_{d, g}^{\prime}(M)$ for some hyperplane $M$ and $C$ is contained in a degree 3 surface $A$ of $M$.

Proof. Since $C$ spans $M$, then $A$ spans $M$. The classification of minimal degree surfaces gives that either $A$ is the Hirzebruch surface $F_{1}$ embedded by the complete linear system $\left|\mathcal{O}_{F_{1}}(h+2 f)\right|$ or it is a cone over a rational normal curve of $\mathbb{P}^{3}$.

(a) Assume that $A$ is the Hirzebruch surface $F_{1}$ embedded by the complete linear system $\left|\mathcal{O}_{F_{1}}(h+2 f)\right|$ and take $a, b$ such that $C \in\left|\mathcal{O}_{F_{1}}(a h+b f)\right|$. Since $C$ has genus $g>0$, we have $b \geq a \geq 2$. We have $d=(a h+b f) \cdot(h+2 f)=a+b$. Since $\omega_{F_{1}} \cong \mathcal{O}_{F_{1}}(-2 h-3 f)$, the adjunction formula gives $2 g-2=(a h+b f)$. $((a-2) h+(b-3) f)=(b-a)(a-2)+a(b-3)$. If $a=2$ and hence 
$b=d-2 \geq 10$, we get $2 g-2 \geq 7$, a contradiction. If $a \geq 3$, we have $2 g-2>4$, a contradiction.

(b) Assume that $A$ is a cone with vertex $o$ over a rational normal curve of $\mathbb{P}^{3}$. Let $u: F_{3} \rightarrow A$ be a minimal desingularization of $A . F_{3}$ is the Hirzebruch surface with the same name with $h=u^{-1}(o)$ and $u$ induced by the complete linear system $\left|\mathcal{O}_{F_{3}}(h+3 f)\right|$. Let $C^{\prime} \subset F_{3}$ be the strict transform of $C$. Take $a, b$ such that $C \in\left|\mathcal{O}_{F_{3}}(a h+b f)\right|$ with $b \geq 3 a$. We have $d=b$. We have $\omega_{F_{3}} \cong \mathcal{O}_{F_{3}}(-2 h-5 f) \mid$ Since $C$ is smooth, $u$ induces an isomorphism of $C^{\prime}$ onto $C$. The adjunction formula gives $2 g-2=(a h+b f) \cdot((a-2) h+(d-5) f)=$ $(a-2)(d-3 a)+a(d-5)$. Since $g>0$, we have $a \geq 2$ and hence $2 \leq a \leq\lfloor d / 3\rfloor$. In all cases we get $2 g-2>4$, a contradiction.

Lemma 13. Fix $C \in M_{d, g}^{\prime}(M)$ and let $H \subset M$ be a general hyperplane. Fix an integer $t \geq 1$.

(a) If $3 t+1 \geq d$, then $h^{1}\left(H, \mathcal{I}_{\mathrm{C} \cap H, H}(t)\right)=0$.

(b) Assume $d \geq 3 t+2$. Then $h^{1}\left(\mathcal{I}_{C \cap H, H}(t)\right) \leq d-3 t-1$ and equality holds only if $C \cap H$ is contained in a rational normal curve of $H$.

(c) If $C$ is contained in a general heptic hypersurface and $C \cap H$ is contained in a rational normal curve of $M$, then $h^{1}\left(\mathcal{I}_{C}(7)\right) \geq 3 d-23($ case $g=1)$ or $h^{1}\left(\mathcal{I}_{C}(7)\right) \geq$ $3 d-16-6 g($ case $g=2,3)$.

Proof. The scheme $C \cap H$ is a set of $d$ points of $H$ spanning $H=\mathbb{P}^{3}$ and in uniform position. Part (a) follows from [10, Theorem 3.2] and part (b) is proved as in Lemma 8.

Now we prove part (c). Let $T \subset H$ be a rational normal curve of $H$ containing $C \cap H$.

(i) First assume $g=1$ and set $Z:=C \cap H$. Since $T$ has $\infty^{d}$ subsets of cardinality $d, \mathbb{P}^{5}$ has $\infty^{8} 3$-dimensional linear spaces, each 3-dimensional $H \subset \mathbb{P}^{5}$ is contained in $\infty^{1}$ hyperplanes of $\mathbb{P}^{5}$ and each 3-dimensional linear space has $\infty^{12}$ rational normal curves we get part (c) for $g=1$ if we prove that $h^{1}\left(N_{C, M}(-Z)\right)=$ 0 , i.e. $h^{1}\left(N_{C, M}(-1)\right)=0$. Since $C$ is a curve, $h^{2}(\mathcal{F})=0$ for all coherent sheaves $\mathcal{F}$ on $C$. Hence it is sufficient to prove that $h^{1}\left(C, T M_{\mid C}(-1)\right)=0$. Assume $h^{1}\left(C, T M_{\mid C}(-1)\right)>0$. By duality there is a non-zero map $T M_{\mid C} \rightarrow \mathcal{O}_{C}(1)$. Fix homogeneous coordinates $z_{0}, z_{1}, z_{2}, z_{3}, z_{4}$ of $M$. The Euler's sequence gives a nonzero map $\mathcal{O}_{C}(1)^{\oplus 5} \rightarrow \mathcal{O}_{C}(1)$, i.e. $\left(a_{0}, a_{1}, a_{2}, a_{5}\right) \in \mathbb{C}^{5} \backslash\{0\}$. Hence $C$ is contained in the hyperplane $\left\{\sum_{i} a_{i} z_{i}=0\right\}$, a contradiction.

(ii) Now assume $g \geq 2$. Set $b:=d+2-2 g$ and take $Z \subset C \cap H$ with $\operatorname{deg}(Z)=b$. Since $T$ has $\infty^{b}$ subsets of cardinality $b, \mathbb{P}^{5}$ has $\infty^{8}$ 3-dimensional linear spaces, each 3-dimensional $H \subset \mathbb{P}^{5}$ is contained in $\infty^{1}$ hyperplanes of $\mathbb{P}^{5}$ and each 3-dimensional linear space has $\infty^{12}$ rational normal curves, we get part (c) for $g=2,3$ if we prove that $h^{1}\left(N_{C, M}(-Z)\right)=0$. Assume $h^{1}\left(N_{C, M}(-Z)\right)>$ 0 . Since $N_{C, M}$ is a quotient of $\mathcal{O}_{C}(1)^{\oplus 5}$, we get $h^{1}\left(\mathcal{O}_{C}(1)(-Z)\right)>0$. Since $\operatorname{deg}\left(\mathcal{O}_{C}(1)(-Z)\right)=2 g-2$, we get $\mathcal{O}_{C}(1)(-Z) \cong \omega_{Z}$. Since $b<d$, there is $Z^{\prime} \subset C \cap H$ with $\sharp\left(Z \cap Z^{\prime}\right)=b-1$. Set $\{p\}:=Z \backslash Z \cap Z^{\prime}$. By monodromy the value of $h^{1}\left(\mathcal{O}_{C}(1)(-A)\right)$ is the same for all $A \subset C \cap H$ with cardinality $b$. Hence $\mathcal{O}_{C}(1)\left(-Z^{\prime}\right) \cong \mathcal{O}_{C}(1)(-Z)$, i.e. $p$ and $q$ are linearly equivalent, contradicting the assumption $g>0$. 
Lemma 14. A general $W \in \mathcal{W}$ contains no $C \in M_{d, g}$ such that $C \in M_{d, g}^{\prime}(M)$ for some hyperplane $M$ and $h^{0}\left(M, \mathcal{I}_{C, M}(2)\right) \geq 3$.

Proof. Fix $C \in M_{d, g}^{\prime}(M)$ such that $h^{0}\left(M, \mathcal{I}_{C, M}(2)\right) \geq 3$. Let $K \subset M$ be the settheoretic base locus of $\left|\mathcal{I}_{C, M}(2)\right|$. Since $C$ spans $M$, every quadric hypersurface of $M$ containing $C$ is irreducible. Hence $\operatorname{dim}(K) \leq 2$. Let $A \subseteq K$ be any irreducible component of $K$ containing $C$. First assume $\operatorname{dim}(A)=2$. Since $C$ spans $M$, then $A$ spans $M$ and hence $\operatorname{deg}(A) \geq 3$. Fix 2 general $Q_{1}, Q_{2}$ elements of $\left|\mathcal{I}_{C, M}(2)\right|$. Since $h^{0}\left(M, \mathcal{I}_{Q_{1} \cap Q_{2}}(2)\right)=2<h^{0}\left(M, \mathcal{I}_{C, M}(2)\right)=h^{0}\left(M, \mathcal{I}_{A, M}(2)\right)$, Bezout gives $A \subsetneq Q_{1} \cap Q_{2}$ and hence $\operatorname{deg}(A)=3$. Use Lemma 12. Now assume $\operatorname{dim}(A)=1$, i.e. $A=C$. Fix general $Q_{1}, Q_{2}, Q_{3}$ and set $T:=Q_{1} \cap Q_{2} \cap Q_{3}$. Since $d>8$, and $C$ is an irreducible component of $T$, the contradiction comes from Bezout ([12, Theorem 2.2.5]).

Lemma 15. Fix $C \in M_{d, g}^{\prime}(M)$ and let $H \subset M$ be a general hyperplane. Set $Z:=C \cap H$.

(i) If $h^{0}\left(H, \mathcal{I}_{Z, H}(2)\right)=0$, then we have $h^{1}\left(H, \mathcal{I}_{Z, H}(3)\right) \leq \max \{0, d-13\}$ and $h^{1}\left(H, \mathcal{I}_{Z, H}(4)\right)=0$.

(ii) If $h^{0}\left(H, \mathcal{I}_{Z, H}(2)\right)=1$, then we have $h^{1}\left(H, \mathcal{I}_{Z, H}(3)\right) \leq \max \{0, d-12\}$, $h^{1}\left(H, \mathcal{I}_{Z, H}(3)\right) \leq 2$ if $d \in\{15,16\}$ and $h^{1}\left(H, \mathcal{I}_{Z, H}(4)\right)=0$.

(iii) If $^{0}\left(H, \mathcal{I}_{Z, H}(2)\right)=2$, then $h^{1}\left(H, \mathcal{I}_{Z, H}(3)\right) \leq d-11, h^{1}\left(H, \mathcal{I}_{Z, H}(3)\right) \leq d-12$ for all $d \geq 13, h^{1}\left(H, \mathcal{I}_{Z, H}(4)\right) \leq \max \{0, d-15\}$ and $Z$ is contained in a complete intersection $T \subset H$ of 2 quadrics; if $d=16$ and $h^{1}\left(H, \mathcal{I}_{Z, H}(4)\right)>0$, then $C$ is not contained in a general heptic hypersurface; if $d=12$ and $h^{1}\left(H, \mathcal{I}_{Z, H}(3)\right)>$ 0 , then $h^{0}\left(M, \mathcal{I}_{C, M}(2)\right)>0$.

(iv) To rule out from a general heptic hypersurface all $C \in M_{d, g}$ such that there is a hyperplane $M \subset \mathbb{P}^{5}$ with $C \in M_{d, g}^{\prime}(M)$ and $h^{0}\left(H, \mathcal{I}_{C \cap H, H}(2)\right)=2$ for a general hyperplane $H$ of $M$ it is sufficient to rule out all $C \in M_{d, g}^{\prime}(M)$ with $h^{1}\left(M, \mathcal{I}_{C, M}(7)\right) \geq 3 d-6 g-21$.

Proof. The set $Z$ is in uniform position in $H$ and it spans $H$. First assume $h^{0}\left(H, \mathcal{I}_{Z, H}(2)\right)=0$. Fix any $S \subset C \cap H$ with $\sharp(S)=9 . \quad S$ is contained in a unique quadric surface $Q_{S}$ and $Q_{S} \cap C \cap H=S$. Fix $p \in C \cap H$ and any $S^{\prime} \subset Z \backslash(S \cup\{p\})$ with $\sharp\left(S^{\prime}\right) \leq 3$. Since $Z$ is in uniform position, there is a plane $N \subset H$ with $N \cap Z=S^{\prime}$. The cubic $Q_{S} \cup N$ shows that $h^{1}\left(H, \mathcal{I}_{S \cup S^{\prime} \cup\{p\}}(3)\right)=$ $h^{1}\left(H, \mathcal{I}_{S \cup S^{\prime}}(3)\right)$. Hence $h^{1}\left(H, \mathcal{I}_{Z, H}(3)\right) \leq \max \{0, d-13\}$. Since $d-10 \leq 9$, we have $h^{0}\left(H, \mathcal{I}_{Z \backslash(S \cup\{p\})}(2)\right)>0$. Take a general $Q^{\prime} \in\left|\mathcal{I}_{Z \backslash(S \cup\{p\}), H}(2)\right|$. Since $Z$ is in uniform position and $\sharp(Z \backslash(S \cup\{p\})) \leq 8$, we have $Q^{\prime} \cap Z=Z \backslash(S \cup\{p\})$. Hence $\left(Q \cup Q^{\prime}\right) \cap Z=Z \backslash\{p\}$. Hence $h^{1}\left(H, \mathcal{I}_{Z \backslash\{p\}, H}(4)\right)=h^{1}\left(H, \mathcal{I}_{Z, H}(4)\right)$. Taking smaller subsets of $Z \backslash(S \cup\{p\})$ we get in finitely steps that $h^{1}\left(H, \mathcal{I}_{Z, H}(4)\right)=$ 0 .

Now assume $h^{0}\left(H, \mathcal{I}_{Z, H}(2)\right)=1$. Take $S_{1} \subset Z$ with $\sharp\left(S_{1}\right)=8$ and let $Q_{1} \subset H$ be a general element of $\left|\mathcal{I}_{S_{1}, H}(2)\right|$. Since $Z$ is in uniform position, we have $h^{1}\left(H, \mathcal{I}_{S_{1}, H}(2)\right)=0$ and $Q_{1} \cap Z=S_{1}$. Taking planes we get $h^{1}\left(H, \mathcal{I}_{Z, H}(3)\right) \leq$ $\max \{0, d-12\}$. Since $d \leq 17$, any $S_{2} \subset Z \backslash S_{1}$ with $\sharp\left(S_{2}\right) \leq 8$ has 
$h^{1}\left(H, \mathcal{I}_{S_{2}, H}(2)\right)=0$ and $Q_{2} \cap Z=S_{2}$ for a general $Q_{2} \in\left|\mathcal{I}_{S_{2}, H}(2)\right|$, we get $h^{1}\left(H, \mathcal{I}_{Z, H}(4)\right)=0$. Now assume $d \in\{15,16\}$. Let $Q \subset H$ be the quadric surface containing $Z$. Since $Z$ is in uniform position, $Q$ is irreducible. Since $h^{0}\left(Q, \mathcal{O}_{Q}(3)\right)=16$, it is sufficient to prove that $h^{0}\left(Q, \mathcal{I}_{Z, Q}(2)\right) \leq 18-d$. In particular we may assume $h^{0}\left(Q, \mathcal{I}_{Z, Q}(3)\right)>0$. Fix a general $D \in\left|\mathcal{I}_{Z, Q}(3)\right|$. Since $Z$ is in uniform position in $Q$ (or by monodromy), $D$ is an integral curve and hence it is a canonically embedded integral curve, which is the complete intersection of $Q$ and a cubic surface. Riemann-Roch gives $h^{0}\left(D, \mathcal{O}_{D}(3)\right)=15$. Since $D$ is arithmetically Cohen-Macaulay, to prove that $h^{1}\left(H, \mathcal{I}_{Z, H}(3)\right) \leq 2$ it is sufficient to prove that $h^{1}\left(D, \mathcal{I}_{Z, D}(3)\right) \leq 2$. Take any zero-dimensional scheme $Z^{\prime} \subset D$ such that $\operatorname{deg}\left(Z^{\prime}\right)=16$ and $Z^{\prime} \supseteq Z$. It is sufficient to prove that $h^{1}\left(D, \mathcal{I}_{Z^{\prime}, D}(3)\right) \leq 2$. This is true (by duality), because the very ampleness of $\omega_{D} \cong \mathcal{O}_{D}(1)$ implies $h^{0}(D, \mathcal{L}) \leq 1$ for every rank 1 torsion free sheaf $\mathcal{L}$ on $D$ with $\operatorname{deg}(\mathcal{L})=2$.

Now assume $h^{0}\left(\mathcal{I}_{Z, H}(2)\right)=2$. Since $Z$ is in uniform position, for all $S_{3} \subset Z$ with $\sharp\left(S_{3}\right) \leq 7$ we have $h^{1}\left(\mathcal{I}_{S_{3}, H}(2)\right)=0$ and $Q_{3} \cap Z=S_{3}$ for a general $Q \in\left|\mathcal{I}_{S_{3}, H}(2)\right|$. As above we get $h^{1}\left(H, \mathcal{I}_{Z, H}(4)\right) \leq \max \{0, d-15\}$ and $h^{1}\left(H, \mathcal{I}_{Z, H}(3)\right) \leq d-11$. Let $T$ be the intersection of all elements of $\left|\mathcal{I}_{Z, H}(2)\right|$. Since $Z$ is in uniform position, all elements of $\left|\mathcal{I}_{Z, H}(2)\right|$ are irreducible. Hence $\operatorname{dim}(T)=1$. Assume for the moment that $T$ is either not reduced or reducible. Since $Z$ is in uniform position and it is a set, there is an irreducible curve $T_{1} \subset T$ with $\operatorname{deg}\left(T_{1}\right) \leq 3$ and $Z \subset T_{1}$. Since $h^{0}\left(H, \mathcal{I}_{T_{1}, H}(2)\right) \geq 3$ and $Z \subset T_{1}$ we get a contradiction. Hence $T$ is an integral degree 4 curve with $p_{a}(T)=1$. By mon-

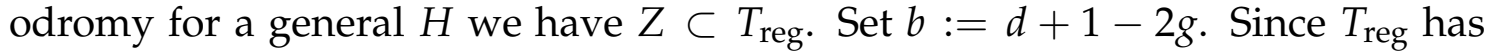
$\infty^{b}$ subsets with cardinality $b, \mathbb{P}^{5}$ has $\infty^{8} 3$-dimensional linear spaces and each $H \in G(3,5)$ has $\infty^{16}$ complete intersections of 2 quadrics, to rule out all $C \in M_{d, g}$ for which there is $M$ and $H$ with $C \in M_{d, g}^{\prime}(M)$ and $C \cap H$ contained in an integral degree 4 complete intersection, it is sufficient to rule out all $C \in M_{d, g}^{\prime}(M)$ with $h^{1}\left(M, \mathcal{I}_{C, M}(7)\right) \geq 3 d-6 g-21$. If $h^{0}\left(M, \mathcal{I}_{C, M}(2)\right)=0$, then $C$ is arithmetically Gorenstein ([17]) and hence $h^{1}\left(M, \mathcal{I}_{C, M}(7)\right)=0$, a contradiction. Since $p_{a}(T)=1$ and $h^{1}\left(H, \mathcal{I}_{T, H}(3)\right)=0$, we have $h^{1}\left(H, \mathcal{I}_{A, H}(3)\right)=0$ for every zero-dimensional scheme $A \subset T$ with either $\operatorname{deg}(A) \leq 11$ or $\operatorname{deg}(A)=12$ and $A \notin\left|\mathcal{O}_{T}(3)\right|$. Hence $h^{1}\left(H, \mathcal{I}_{Z, H}(3)\right)=0$ if $d \leq 11$. Assume $d=12$ and $h^{1}\left(H, \mathcal{I}_{Z, H}(3)\right)>0$. We get $Z \in \mathcal{O}_{T}(3) \mid$ and we conclude quoting [17]. Assume $d \geq 13$. Take $A_{1}, A_{2} \subset C \cap H$ such that $\sharp\left(A_{1}\right)=\sharp\left(A_{2}\right)=12$ and $\sharp\left(A_{1} \cap A_{2}\right)=11$. Set $\{p\}:=A_{1} \backslash A_{1} \cap A_{2}$ and $\{q\}=A_{2} \backslash A_{1}$. By monodromy we have $h^{1}\left(H, \mathcal{I}_{A_{1}, H}(3)\right)=h^{1}\left(H, \mathcal{I}_{A_{2}, H}(3)\right)$, i.e. $h^{1}\left(T, \mathcal{I}_{A_{1}, T}(3)\right)=h^{1}\left(T, \mathcal{I}_{A_{2}, T}(3)\right)$. Assume $h^{1}\left(T, \mathcal{I}_{A_{i}, T}(3)\right)>0$, i.e. $A_{i} \in\left|\mathcal{O}_{T}(3)\right|$. We get that $p$ and $q$ are linear equivalent, contradicting the inequality $p_{a}(T)>0$.

Now assume $d=16$ and $h^{1}\left(H, \mathcal{I}_{C \cap H, T}(4)\right)>0$. Since $\omega_{T} \cong \mathcal{O}_{T}$, we have $C \cap H \in\left|\mathcal{O}_{T}(4)\right|$, i.e. (since $\left.h^{1}\left(H, \mathcal{I}_{T}(4)\right)=0\right) C \cap H$ is the complete intersection of a quartic and 2 quadrics. By [33, Theorem 0.1] we have $d \equiv 2(\bmod 3)$ and that $C \cap H$ is contained in rational normal curve; both statements are false.

Lemma 16. Fix $C \in M_{d, g}^{\prime}(M), d \geq 13$, such that $\alpha(C)=3$ and $h^{0}\left(M, \mathcal{I}_{C, M}(3)\right) \geq 2$. Let $H \subset M$ be a general hyperplane. Then

(a) $\mathrm{C} \cap \mathrm{H}$ is not contained in a curve of degree $\leq 3$ or degree 4 and arithmetic genus 1. 
(b) $h^{1}\left(H, \mathcal{I}_{C \cap H, H}(4)\right)=0, h^{1}\left(H, \mathcal{I}_{Z, H}(3)\right) \leq \max \{0, d-12\}$, $h^{1}\left(H, \mathcal{I}_{Z, H}(3)\right) \leq 2$ if $d=15$ and $h^{1}\left(H, \mathcal{I}_{Z, H}(3)\right) \leq 3$ if $d=\overline{16}$.

Proof. Let $K \subset M$ be the set-theoretic base locus of $\left|\mathcal{I}_{C, M}(3)\right|$. Since $\alpha(C)=3$ and $h^{0}\left(M, \mathcal{I}_{C, M}(3)\right) \geq 2$, we have $\operatorname{dim}(K) \leq 2$. Assume that for a general $H \subset M$, the set $C \cap H$ is contained in a curve $T_{H}$ with $c:=\operatorname{deg}\left(T_{H}\right) \leq 4$ and $c$ minimal. Since $H$ is general, $C \cap H$ is a set of $d$ points of $H$ spanning $H$ and in uniform position. Since $d>c$, monodromy and the minimality of $c$ gives that $T_{H}$ is irreducible. Since $d>12$, Bezout's theorem gives $T_{H} \subset K \cap H$. Hence there is an irreducible component $A$ of $K$ with $\operatorname{dim}(A)=2$ and $T_{H} \subset A \cap H$ for a general hyperplane $H$. For a general $H, A \cap H$ is irreducible. Since $T_{H} \subseteq A \cap H$, we get $T_{H}=A \cap H$ and hence $\operatorname{deg}(A)=c$. Lemma 12 gives $c=4$. Assume $p_{a}\left(T_{H}\right)=1$, i.e. assume that $T_{H}$ is the complete intersection of 2 quadrics. Since $h^{i}\left(H, \mathcal{I}_{T_{H}}(1)\right)=0, i=0,1$, and $A \cap H=T_{H}$, the exact sequence (3) gives that $A$ is a complete intersection of 2 quadric hypersurfaces of $M$. Since $C \subset A$, we get $\alpha(C)=2$, a contradiction. Now we prove part (b). By part (a) we have $h^{0}\left(H, \mathcal{I}_{C \cap H, H}(2)\right) \leq 1$. Use parts (i) and (ii) of Lemma 17.

Lemma 17. Let $\Gamma$ be the set of all $C \in M_{d, g}$ such that there is a hyperplane $M \subset \mathbb{P}^{5}$ with $C \in M_{d, g}^{\prime}(M)$ and $h^{0}\left(M, \mathcal{I}_{C, M}(2)\right)>0$. Then $\operatorname{dim}(\Gamma) \leq 3 d+19+g$.

Proof. Since $\mathbb{P}^{5}$ has $\infty^{5}$ hyperplanes, it is sufficient to prove that for each hyperplane $M$ the set $\Psi$ of all $C \in M_{d, g}^{\prime}(M)$ and $h^{0}\left(M, \mathcal{I}_{C, M}(2)\right)>0$ has dimension $\leq 3 d+14+g$. Fix $C \in \Psi$ and let $Q \subset M$ be any quadric containing $C$. Since $C$ spans $M, Q$ is irreducible. The Hilbert scheme $\operatorname{Hilb}(Q)$ of $Q$ has $H^{0}\left(N_{C, Q}\right)$ as its tangent space at $C$. Let $\tau$ be the tangent sheaf of $Q$.

(a) First assume that either $Q$ is smooth or $C$ does not intersect the singular locus $V$ of $C$. Since $\operatorname{dim}\left|\mathcal{O}_{M}(2)\right|=14$, to handle this case it is sufficient to prove that $h^{0}\left(N_{C, Q}\right) \leq 3 d+g$. Since the algebraic group $\operatorname{Aut}(Q)$ acts transitively on $Q \backslash V$ and $H^{0}(\tau)$ is the tangent space to $\operatorname{Aut}(Q)$ at the identity, $H^{0}(\tau)$ spans $\tau$ at each point of $Q \backslash V$. Since $C \subset Q \backslash V, N_{C, Q}$ is a quotient of $\tau_{\mid C}$ and hence it is spanned. Since $\omega_{Q} \cong \mathcal{O}_{Q}(-3), N_{C, Q}$ is a rank 2 vector bundle with degree $3 d+2 g-2$. Hence $h^{1}\left(\operatorname{det}\left(N_{C, Q}\right)\right)=0$. Since $N_{C, Q}$ is spanned, it is an extension of $\operatorname{det}\left(N_{C, Q}\right)$ by $\mathcal{O}_{C}$ and hence $h^{1}\left(N_{C, Q}\right) \leq g$. Riemann-Roch gives $h^{0}\left(N_{C, Q}\right) \leq$ $3 d+g$.

(b) Now assume $C \cap V \neq \varnothing$ and set $x:=\operatorname{deg}(C \cap V)$. Since $C$ is smooth, $x=1$ if $\operatorname{dim}(V)=0$. By step (a) and the fact that $M$ has $\infty^{13}$ singular quadrics it is sufficient to prove that $h^{0}\left(N_{C, Q}\right) \leq 3 d+1+g$. The vector space $H^{0}(\tau)$ is the tangent space at the identity map of the automorphism group $\operatorname{Aut}(Q)$. Since $Q \backslash V$ is homogeneous, $\tau_{\mid Q \backslash V}$ is a spanned vector bundle. Since $C$ is not a line and $\operatorname{dim}(V) \leq 1$, the set $V \cap C$ is finite. Dualizing the natural map from the conormal sheaf of $C$ in $Q$ to $\Omega_{Q}^{1}$ we get a map $w: \tau_{\mid C} \rightarrow N_{C, Q}$ which is surjective outside the finite set $C \cap V$. Let $u: \widetilde{Q} \rightarrow Q$ be the blowing up of $V$, $E:=u^{-1}(V)$ the exceptional divisor, and $\widetilde{C} \subset \widetilde{Q}$ the strict transform of $C$. Since $C$ is smooth, $u$ maps isomorphically $\widetilde{C}$ onto $C$. Let $\Psi$ be closure in $\operatorname{Hilb}(\widetilde{Q})$ of the strict transforms of all $C \subset Q$ with $\operatorname{deg}(C \cap V)=x$. It is sufficient to prove that $\operatorname{dim}(\Psi) \leq 3 d+1+g$. Take a general $D \in \Psi$. Since $\operatorname{Aut}(\widetilde{Q})$ acts transitively of 
$\widetilde{Q} \backslash E$, step (a) of the proof gives $h^{1}\left(N_{D, \widetilde{Q}}\right) \leq g$. Hence it is sufficient to prove that $\operatorname{deg}\left(N_{D, \widetilde{Q}}\right) \leq 3 d+2 g-1$, i.e. $\operatorname{deg}\left(\tau_{\widetilde{Q} \mid D}\right) \leq 3 d+1$, i.e. $\operatorname{deg}\left(\omega_{\widetilde{Q}} \mid D\right) \geq-3 d-1$.

The group $\operatorname{Pic}(\widetilde{Q})$ is freely generated by $E$ and the pull-back $H$ of $\mathcal{O}_{Q}(1)$. We have $D \cdot H=d$ and $D \cdot E=x$. We have $\omega_{\widetilde{Q}} \cong \mathcal{O}_{\widetilde{Q}}(-3 H+c E)$ with $c=-1$ if $\operatorname{dim}(V)=0$ ([18], Example $8.5(2))$ and $c=0$ if $\operatorname{dim}(V)=1$ ([18], Example 8.5 (3)). Hence $\operatorname{deg}\left(\omega_{\widetilde{Q} \mid \widetilde{C}}\right)=-3 d+c x \geq-3 d-1$ and the proof is complete.

Lemma 18. A general heptic hypersurface contains no $C \in M_{d, g}$ such that there is a hyperplane $M \subset \mathbb{P}^{5}$ with $C \in M_{d, g}^{\prime}(M)$, there is no line $L \subset M$ with $\operatorname{deg}(L \cap C) \geq 6$, no conic $D \subset M$ with $\operatorname{deg}(D \cap C) \geq 12$.

Proof. By [11, Corollaire 2], Lemma 11 and the assumptions on the lines and the conics of $C$, for any integer $t \geq 5$ and any plane $N \subset M$ we have $h^{1}\left(N, \mathcal{I}_{C \cap N, N}(t)\right)=0$. Lemma 1 and the case $r=4$ and $t=5,6,7$ of Lemma 3 give $h^{1}\left(M, \mathcal{I}_{C, M}(4)\right) \geq h^{1}\left(M, \mathcal{I}_{C, M}(7)\right)+12 \geq d+7-g$. Let $H \subset M$ be a general hyperplane.

(a) Assume that the set $H \cap C$ is not contained in a rational normal curve of $H$. By Lemma 15 (assuming that $C$ is contained in a general heptic hypersurface) $h^{1}\left(H, \mathcal{I}_{\mathrm{C} \cap H, H}(4)\right)=0$. Set $\beta=0$ if $d \leq 15$ and $\beta=1$ if $d=16$. By Lemma 15 we have $h^{1}\left(H, \mathcal{I}_{C, H}(3)\right) \leq 1$ if $d=12$ and $h^{1}\left(H, \mathcal{I}_{C \cap H, H}(3)\right) \leq d-12$ if $d \geq 13$. Hence $h^{1}\left(H, \mathcal{I}_{\mathrm{C} \cap H, H}(4)\right)+h^{1}\left(H, \mathcal{I}_{\mathrm{C} \cap H, H}(3)\right) \leq 3+\beta$. By (1) for a general hyperplane $H \subset M$ we have $h^{1}\left(M, \mathcal{I}_{C, M}(2)\right) \geq h^{1}\left(M, \mathcal{I}_{C, M}(4)\right)-3-\beta \geq d+4-$ $g-\beta$, i.e. $h^{0}\left(M, \mathcal{I}_{C, M}(2)\right) \geq 18-d-\beta$. If $d \leq 15$, then we conclude by Lemma 14 . Now assume $d=16$. We got $h^{0}\left(M, \mathcal{I}_{C, M}(2)\right)>0$. By Lemma 17 we may assume $h^{1}\left(M, \mathcal{I}_{C, M}(7)\right) \geq 3 d-17-3 g=21-4 g$. We first get $h^{1}\left(M, \mathcal{I}_{C, M}(4)\right) \geq 33-3 g$ and then $h^{1}\left(M, \mathcal{I}_{C, M}(2)\right) \geq 29-3 g$, i.e. $h^{0}\left(M, \mathcal{I}_{C, M}(2)\right) \geq 10-2 g \geq 4$, contradicting Lemma 14.

(b) Now assume that $C \cap H$ is contained in a rational normal curve $E_{H}$ of $H$. By part (c) of Lemma 13 we may assume that $h^{1}\left(M, \mathcal{I}_{C, M}(7)\right) \geq 3 d-16-6 g-\epsilon$ with $\epsilon=1$ if $g=1$ and $\epsilon=0$ if $g \in\{2,3\}$. We first get $h^{1}\left(M, \mathcal{I}_{C, M}(4)\right) \geq 3 d-$ $4-6 g-\epsilon$ and then $h^{1}\left(M, \mathcal{I}_{C, M}(2)\right) \geq 3 d-4-6 g-\epsilon-\min \{d-13,0\}-d+10$, i.e. $h^{0}\left(M, \mathcal{I}_{C, M}(2)\right) \geq 20-5 g-\epsilon-\min \{d-13,0\}$. Lemma 14 concludes unless $(d, g)=(16,3)$. Assume $(d, g)=(16,3)$. We just proved that $h^{0}\left(M, \mathcal{I}_{C, M}(2)\right) \geq 2$. By Lemma 14 we may assume that $h^{0}\left(M, \mathcal{I}_{C, M}(2)\right)=2$. Let $\Sigma$ the intersection of two different quadric hypersurfaces of $M$ containing $C$. Since $C$ is integral and $C$ is not contained in a degree 3 surface (Lemma 12), $\Sigma$ is an integral complete intersection surface. Hence for a general hyperplane $H \subset M$, the scheme $F_{H}:=$ $H \cap \Sigma$ is an integral complete intersection of two quadrics. By Bezout we have $\sharp\left(E_{H} \cap F_{H}\right) \leq 6$, contradicting the inclusion of $C \cap H$ in $E_{H} \cap F_{H}$.

Notation 2. Fix $d, g$. For any integer $a \geq 0$ let $\mathcal{F}_{a}$ (resp. $\mathcal{G}_{a}$ ) denote the set of all $C \in M_{d, g}$ such that there is a hyperplane $M \subset \mathbb{P}^{5}$ and a line $L \subset M$ (resp. a smooth conic $L \subset M)$ with $C \in M_{d, g}^{\prime}$ and $\operatorname{deg}(L \cap C)=a$. Set $\mathcal{F}_{a}^{\prime \prime}:=\cup_{b \geq a} \mathcal{F}_{b}$ and $\mathcal{G}_{a}^{\prime \prime}:=\cup_{b \geq a} \mathcal{G}_{b}$.

The proof of Lemma 2 gives the following result. 
Lemma 19. Fix a hyperplane $M \subset \mathbb{P}^{5}$ and an integer $b \geq 4$ such that $d \geq b+2 g-$ 1. Let $Z \subset M$ be a zero-dimensional scheme with $\operatorname{deg}(Z)=b$. Then the set of all $C \in M_{d, g}^{\prime}(M)$ containing $Z$ has codimension at least $3 b$ in $M_{d, g}^{\prime}(M)$.

Lemma 20. Fix $d, g$ with $12 \leq d \leq 16$ and $1 \leq g \leq 3$. For any integer $a \geq 6$ set $u_{a}:=\min \{a, d+1-2 g\}$.

(i) Every irreducible component of $\mathcal{F}_{a}^{\prime \prime}, a \geq 6$, has dimension $\leq 5 d+12-g-2 u_{a}$. $2 u_{a}$.

(ii) Every irreducible component of $\mathcal{G}_{a}^{\prime \prime}, a \geq 12$, has dimension $\leq 5 d+17-g-$

Proof. Fix a hyperplane $M \subset \mathbb{P}^{5}$. Set $\mathcal{F}_{a}(M):=\left\{C \in \mathcal{F}_{a}: C \subset M\right\}, \mathcal{G}_{a}(M):=$ $\left\{C \in \mathcal{G}_{a}: C \subset M\right\}, \mathcal{F}_{a}^{\prime \prime}(M):=\cup_{b \geq a} \mathcal{F}_{b}(M)$ and $\mathcal{G}_{a}^{\prime \prime}(M):=\cup_{b \geq a} \mathcal{G}_{b}(M)$. Since in the definitions of $\mathcal{F}_{a}$ and $\mathcal{G}_{a}$ we prescribed that $C \in M_{d, g}^{\prime}(M)$, every $C \in \mathcal{G}_{a}^{\prime \prime} \cup$ $\mathcal{F}_{a}^{\prime \prime}$ is contained in a unique hyperplane. Hence to prove the lemma we may fix a hyperplane $M$, give an upper bound for $\operatorname{dim}\left(\mathcal{F}_{a}^{\prime \prime}(M)\right)$ and $\operatorname{dim}\left(\mathcal{G}_{a}^{\prime \prime}(M)\right)$ and then add +5 to that upper bound. Since the upper bounds in (i) and (ii) are non-decreasing functions of $a$ to prove part (i) (resp. part (ii)) it is sufficient to give a suitable upper bound for all $\mathcal{F}_{a}(M), a \geq 6$, and all $\mathcal{G}_{a}(M), a \geq 12$. Fix $C \in \mathcal{F}_{a}(M)$ (resp. $C \in \mathcal{G}_{a}(M)$ ) and a line $L \subset M$ (resp. a smooth conic $L \subset M$ ) with $\operatorname{deg}(L \cap C)=a$. Fix a zero-dimensional scheme $Z \subseteq C \cap Z$ with $\operatorname{deg}(Z)=$ $u_{a}$. Use Lemma 19, that the smooth curve $L$ has $\infty^{u_{a}}$ subschemes of degree $u_{a}$, that $M$ has $\infty^{6}$ lines and that $M$ has $\infty^{11}$ conics.

Lemma 21. A general heptic hypersurface contain no $C \in M_{d, g}$ such that there is a hyperplane $M$ with $C \in M_{d, g}^{\prime}(M)$ and either there is a line $L \subset M$ with $\operatorname{deg}(C \cap L) \geq 6$ or there is conic $D \subset M$ with $\operatorname{deg}(D \cap C) \geq 12$.

Proof. By Lemma 11 and [11, Corollaire 2] we have $h^{1}\left(N, \mathcal{I}_{C \cap N, N}(t)\right)=0$ if $t \geq 5$ and the plane $N$ contains neither a line $R$ with $\operatorname{deg}(R \cap C) \geq t+2$ nor a conic $D$ with $\operatorname{deg}(D \cap C) \geq 2 t+2$. Until step (e) we assume that either $\alpha(C) \neq 2$ or $d \leq 12$.

(a) We first exclude (with the silent assumption $\alpha(C) \neq 2$ ) all $C \in M_{d, g}$ such that there is a hyperplane $M$ and a line $L \subset M$ with $C \in M_{d, g}^{\prime}(M)$ and $\operatorname{deg}(C \cap L) \geq 9$. Fix $C, L$ such that $\operatorname{deg}(C \cap L) \geq 9$. Set $u_{9}:=\min \{9, d+1-2 g\}$. By Lemma 20 we may assume $h^{1}\left(M, \mathcal{I}_{C, M}(7)\right) \geq d-10-g+2 u_{9}$. Part (a) of Lemma 13 for $t=5,6,7$ gives $h^{1}\left(M, \mathcal{I}_{C, M}(4)\right) \geq \bar{h}^{1}\left(M, \mathcal{I}_{C, M}(7)\right)$.

First assume $d \leq 12$ and so $u_{9}=d+1-2 g$. Lemma 13 gives $h^{1}\left(M, \mathcal{I}_{C, M}(3)\right) \geq$ $h^{1}\left(M, \mathcal{I}_{C, M}(4)\right)$ and $h^{1}\left(M, \mathcal{I}_{C, M}(2)\right) \geq h^{1}\left(M, \mathcal{I}_{C, M}(3)\right)-d+10$. Hence we have $h^{0}\left(M, \mathcal{I}_{C, M}(2)\right) \geq 14-2 d+g+3 d+3-6 g-2=15+d-5 g>0$, a contradiction.

Now assume $d \geq 13$. Note that $u_{9} \geq 8$ and hence $h^{1}\left(M, \mathcal{I}_{C, M}(4)\right) \geq d+6-g$. We get $h^{1}\left(M, \mathcal{I}_{C, M}(3)\right) \geq d+3-g$ (Lemma 12) and hence $h^{0}\left(M, \bar{I}_{C, M}(3)\right) \geq$ $39-2 d-1 \geq 2$, then $\alpha(C)=3$ and then (by Lemma 16) $h^{1}\left(M, \mathcal{I}_{C, M}(3)\right) \geq$ $h^{1}\left(M, \mathcal{I}_{C, M}(4)\right) \geq d+6-g$ and $h^{1}\left(M, \mathcal{I}_{C, M}(2)\right) \geq d+3-g$, i.e. $h^{0}\left(M, \mathcal{I}_{C, M}(2)\right) \geq$ $17-d>0$, a contradiction.

(b) Now we exclude all $C \in M_{d, g}$ such that there is a hyperplane $M$ and a line $L \subset M$ with $C \in M_{d, g}(M)$ and $\operatorname{deg}(C \cap L) \geq 6$. Fix $C, L$ such that $\operatorname{deg}(C \cap L) \geq 6$. Since $d \geq 2 g-1+6$, by Lemma 20 we may assume 
$h^{1}\left(M, \mathcal{I}_{C, M}(7)\right) \geq d+2-g$. By step (a) we may assume that $\operatorname{deg}(R \cap C) \leq 8$ for all lines $R$. Lemma 1 and the case $t=7$ of Lemma 13 give $h^{1}\left(M, \mathcal{I}_{C, M}(4)\right) \geq$ $h^{1}\left(M, \mathcal{I}_{C, M}(6)\right) \geq d+6-g$. We solved this case in step (a).

(c) Now we exclude all $C$ such that there is a conic $D$ with $\operatorname{deg}(D \cap C) \geq 12$ (they have $d \geq 13$ ). By step (b) we may assume $\operatorname{deg}(R \cap C) \leq 5$ for all lines $R$. Thus $D$ is smooth. Set $b:=\min \{12, d+1-2 g\}$. By Lemma 20 we may assume that $h^{1}\left(M, \mathcal{I}_{C, M}(7)\right) \geq d-15-g+2 b$. By Lemma 1 and the case $t=7$ of Lemma 13 we may assume $h^{1}\left(M, \mathcal{I}_{C, M}(6)\right) \geq d-11-g+2 b$. Since $2 b \geq 17$, we conclude as in steps (a) and (b).

(d) Now we assume $d \geq 13$ and $\alpha(C)=2$. By Lemma 17 we may assume $h^{1}\left(M, \mathcal{I}_{C, M}(7)\right) \geq 3 d-17-3 g$.

(d1) First assume that there is no line $L$ with $\operatorname{deg}(L \cap C) \geq 9$. By Lemmas 1 and 13 (case $t=7$ ), we have $h^{1}\left(M, \mathcal{I}_{C, M}(6)\right) \geq h^{1}\left(\mathcal{I}_{C, M}(7)\right)+4 \geq 3 d-13-3 g$. Part (a) of Lemma 13 for $t=5,6$ gives $h^{1}\left(M, \mathcal{I}_{C, M}(4)\right) \geq 3 d-13-3 g$. The cases $t=3,4$ of Lemma 13 gives $h^{1}\left(M, \mathcal{I}_{C, M}(2)\right) \geq 3 d-13-3 g+10-d-$ $\min \{0, d-13\}$, i.e. $h^{0}\left(M, \mathcal{I}_{C, M}(2)\right) \geq 11-2 g-\min \{0, d-13\}$. Lemma 14 gives $d=16, g=3$ and $h^{0}\left(M, \mathcal{I}_{C, M}(2)\right)=2$. Let $K$ be the intersection of 2 different elements of $\left|\mathcal{I}_{C, M}(2)\right| . K$ is a degree 4 surface and for a general hyperplane $H$ of $M K \cap H$ is an integral degree 4 curve, which is the complete intersection of 2 quadrics. Hence for a general $H$ the set $C \cap H$ is not contained in a rational normal curve of $H$. Hence $h^{1}\left(M, \mathcal{I}_{C, M}(3)\right) \geq h^{1}\left(M, \mathcal{I}_{C, M}(4)\right)-2$ and $h^{1}\left(M, \mathcal{I}_{C, M}(2)\right) \geq h^{1}\left(M, \mathcal{I}_{C, M}(3)\right)-4$ (Lemma 13). Hence $h^{1}\left(M, \mathcal{I}_{C, M}(2)\right) \geq 20$, i.e. $h^{0}\left(M, \mathcal{I}_{C, M}(2)\right) \geq 5$, a contradiction.

(d2) Now assume the existence of a line $L \subset M$ such that $e:=\operatorname{deg}(L \cap C) \geq$ 9. Let $\mathcal{U}$ be the set of all lines $R \subset M$ such that $\operatorname{deg}(R \cap C) \geq 7$ and let $\mathcal{V}$ be the set of all planes spanned by conics $D$ such that $\operatorname{deg}(D \cap C) \geq 12$.

(d2.1) Assume for the moment that $\mathcal{U} \cup \mathcal{V}$ is finite. Let $N \subset M$ be a general plane. We have $N \cap R=\varnothing$ for all $R \in \mathcal{U}$ and $N \cap A$ is a single point for every $A \in \mathcal{V}$. Let $V \subset H^{0}\left(\mathcal{O}_{M}(1)\right)$ be the 2-dimensional linear subspace parametrizing all hyperplanes $U$ of $M$ containing $N$. For any such $U$ we have $R \nsubseteq U$ for all $R \in \mathcal{U}$ and $\operatorname{dim}(U \cap A)=1$ for each $A \in \mathcal{V}$. Hence $U$ contains no line $R$ with $\operatorname{deg}(R \cap C) \geq 7$ and no conic $D$ with $\operatorname{deg}(C \cap D) \geq 12$. By Lemma 11 and [11, Corollaire 2] we have $h^{1}\left(A, \mathcal{I}_{C \cap A, A}(5)\right)=0$ for every plane $A \subset U$ and then Lemma 3 for $r=3, t=5$ and $c \leq 16$ gives $h^{1}\left(U, \mathcal{I}_{C \cap U, U}(5)\right)=0$. Lemma 1 for $t=5,6,7$ gives $h^{1}\left(M, \mathcal{I}_{C, M}(4)\right) \geq h^{1}\left(M, \mathcal{I}_{C, M}(7)\right)+3$. As in step (d1) we get $15 \leq d \leq 16$ and $g=3$. As in step (d1) we exclude the case $h^{0}\left(M, \mathcal{I}_{C, M}(2)\right)=2$ (and in particular the case $d=15$ ), i.e. we may assume $d=16$ and $h^{0}\left(M, \mathcal{I}_{C, M}(2)\right)=1$. Let $Q \subset M$ denote the quadric containing $C$. We have $h^{1}\left(M, \mathcal{I}_{C, M}(3)\right) \geq h^{1}\left(M, \mathcal{I}_{C, M}(4)\right)-3 \geq 22$, i.e. $h^{0}\left(M, \mathcal{I}_{C, M}(3)\right) \geq 35-46+22=$ $11 \geq 7$. Hence $h^{0}\left(Q, \mathcal{I}_{C, Q}(3)\right) \geq 2$. Fix two general $U_{1}, U_{2} \in\left|\mathcal{I}_{C, Q}(3)\right|$ and set $K:=U_{1} \cap U_{2} . K$ is the complete intersection in $M$ of a quadric and 2 cubics, because any element of $\left|\mathcal{I}_{C, Q}(3)\right|$ is an irreducible surface, since $h^{0}\left(Q, \mathcal{I}_{C, Q}(2)\right)=$ $h^{0}\left(M, \mathcal{I}_{C, M}(2)\right)-1=0$. The complete intersection $K$ links $C$ to a degree 2 locally Cohen-Macaulay curve $E$. By Bezout we have $L \subset K$ and so either $E$ is a double structure on $L$ (a rope) or it is the union of $L$ and another line $R$. Set $R:=L$ if $E$ is a rope. The complete intersection $K$ links $R$ to the curve $C \cup L$. Since $R$ is arithmetically Cohen-Macaulay, $C \cup L$ is arithmetically Cohen-Macaulay. In particular 
$h^{1}\left(M, \mathcal{I}_{C \cup L, M}(7)\right)=0$. Since $h^{1}\left(M, \mathcal{I}_{C, M}(7)\right) \geq 3 d-14-3 g>d \geq \operatorname{deg}(C \cap L)$, we got a contradiction.

(d2.2) Now assume that $\mathcal{U}$ is infinite. Take $R \in \mathcal{U}$ with $R \neq L$. Assume $R \cap L \neq \varnothing$. Since $\operatorname{deg}(L \cap R)=1$, we get $\operatorname{deg}(L \cap C)=9, \operatorname{deg}(R \cap C)=7 L \cap R \in$ $C$. The plane $A$ spanned by $R \cup L$ has $\operatorname{deg}(A \cap C) \geq 15$, contradicting Lemma 11. Now assume $R \cap L=\varnothing$. The hyperplane $H$ spanned by $R \cup L$ intersects $C$ in a scheme of degree $\geq 16$. We get $d=16, \operatorname{deg}(L \cap C)=9$ and $\operatorname{deg}(R \cap C)=7$. Since $C$ is smooth, the linear projection $M \backslash L \rightarrow \mathbb{P}^{2}$ from $L$ induces a morphism $u: C \rightarrow \mathbb{P}^{2}$ such that $\operatorname{deg}(u) \cdot \operatorname{deg}(u(C)=7$. Since there are infinitely many $R \in \mathcal{U}$, we get $\operatorname{deg}(u) \geq 7$ and so $u(C)$ is a line, contradicting the assumption $C \in M_{d, g}^{\prime}(M)$.

(d2.3) Now assume that $\mathcal{V}$ is infinite. Let $\mathcal{V}^{\prime}$ be an irreducible component of $\mathcal{V}$ with $\operatorname{dim}\left(\mathcal{V}^{\prime}\right)>0$. Assume the existence of a conic $D \subset M$ such that $\operatorname{deg}(D \cap C) \geq 12$ and call $A$ the plane spanned by $D$. If $A \cap L$ is a point, then the 3-dimensional linear space spanned by $A \cup L$ intersects $C$ in a scheme of degree $\geq 12+9-1>d$, a contradiction.

Now assume $L \subset A$ for infinitely many planes $A \in \mathcal{V}^{\prime}$. For any plane $N \subset M$ with $N \cap L=\varnothing$, the set $N \cap A$ is a single point. Hence as in step (d2.1) we may ignore all these planes $A \in \mathcal{V}^{\prime}$, even if infinite.

Now take a general $A \in \mathcal{V}^{\prime}$ and assume $A \cap L=\varnothing$ and $f:=\operatorname{deg}(D \cap C)$ with $D \subset A$ a conic and $f \geq 12$. Take general $A_{1}, A_{2} \in \mathcal{V}^{\prime}$ and let $D_{i} \subset A_{i}, i=1,2$, be the conic with $\operatorname{deg}\left(D_{i} \cap C\right)=f$. First assume that $A_{1} \cap A_{2}$ is a line (this is the case if either $D_{1}$ and $D_{2}$ have a common component or $D_{1} \cap D_{2}$ is a zero-dimensional scheme of degree $\geq 2$ ). We get that either all $A \in \mathcal{V}^{\prime}$ are contained in a fixed hyperplane of $M$ (absurd, because $C$ spans $M$ ) or there is a line $R \subset M$ contained in all $A \in \mathcal{V}^{\prime}$. If $N \subset M$ is a plane with $R \cap N=\varnothing$, then no conic of some $A \in \mathcal{V}^{\prime}$ is contained in a hyperplane of $M$ containing $N$.

(d2.3.1) Assume $D_{1} \cap D_{2}=\varnothing$. We have $h^{0}\left(\mathcal{O}_{D_{1} \cup D_{2} \cup L}(2)\right)=13$ and therefore we have $h^{0}\left(M, \mathcal{I}_{D_{1} \cup D_{2} \cup L}(2)\right) \geq 2$. Fix any $Q^{\prime} \in\left|\mathcal{I}_{D_{1} \cup D_{2} \cup L}(2)\right|$. Since $\operatorname{deg}\left(C \cap Q^{\prime}\right) \geq 2 f+e \geq 33$, Bezout gives $C \subset Q^{\prime}$. Hence $h^{0}\left(M, \mathcal{I}_{C, M}(2)\right) \geq 2$. By Lemma 14 we may assume $h^{0}\left(M, \mathcal{I}_{C, M}(2)\right)=2$ and call $K^{\prime}$ the degree 4 irreducible surface which is the base locus of $\left|\mathcal{I}_{C, M}(2)\right|$. For a general hyperplane $H \subset M$ the scheme $K^{\prime} \cap H$ is an integral curve complete intersection of 2 quadrics and containing $C \cap H$. By Bezout $C \cap H$ is not contained in a rational normal curve of $H$ and so $h^{0}\left(H, \mathcal{I}_{C \cap H, H}(2)\right)=2$. Lemma 15 gives $h^{1}\left(M, \mathcal{I}_{C, M}(3)\right) \geq$ $h^{1}\left(M, \mathcal{I}_{C, M}(4)\right) \geq 3 d-17-3 g$, i.e. $h^{0}\left(M, \mathcal{I}_{C, M}(3)\right) \geq 17-2 g>10$. Hence the map $H^{0}\left(M, \mathcal{I}_{C, M}(2)\right) \otimes H^{0}\left(M, \mathcal{O}_{M}(1)\right) \rightarrow H^{0}\left(M, \mathcal{I}_{C, M}(3)\right)$ is not surjective. Therefore $C$ is contained in the intersection of $K^{\prime}$ with a cubic hypersurface, contradicting Bezout and the inequality $d>12$.

(d2.3.2) Now assume that the scheme $D_{1} \cap D_{2}$ is a single point. First assume that either $e \geq 10$ or $f \geq 13$ or $d \leq 15$ or $D_{1} \cap D_{2} \notin C$. Since $h^{0}\left(\mathcal{O}_{D_{1} \cup D_{2} \cup L}(2)\right) \leq$ 12 , we have $h^{0}\left(M, \mathcal{I}_{D_{1} \cup D_{2} \cup L}(2)\right) \geq 3$. Fix any $Q^{\prime} \in\left|\mathcal{I}_{D_{1} \cup D_{2} \cup L}(2)\right|$. Since $\operatorname{deg}\left(C \cap Q^{\prime}\right) \geq 2 f+e-\operatorname{deg}\left(D_{1} \cap D_{2} \cap C\right) \geq 2 d+1$, Bezout gives $Q^{\prime} \supset C$, contradicting Lemma 14. Now assume $e=9, f=12, d=16$ and that $D_{1} \cap D_{2}$ is a point $p \in C$. Take a general $q \in C$. Since $h^{0}\left(\mathcal{O}_{D_{1} \cup D_{2} \cup L \cup\{q\}}(2)\right) \leq 13$, we have $h^{0}\left(M, \mathcal{I}_{D_{1} \cup D_{2} \cup L}(2)\right) \geq 2$. Fix any $Q^{\prime} \in\left|\mathcal{I}_{D_{1} \cup D_{2} \cup L}(2)\right|$. Since $\operatorname{deg}\left(C \cap Q^{\prime}\right) \geq$ 
$2 f-1+e-\operatorname{deg}\left(D_{1} \cap D_{2} \cap C\right)+1 \geq 2 d+1$, then $Q^{\prime} \supset C$. Hence $h^{0}\left(M, \mathcal{I}_{C, M}(2)\right) \geq$ 2. By Lemma 14 we may assume $h^{0}\left(M, \mathcal{I}_{C, M}(2)\right)=2$ and call $K^{\prime}$ the degree 4 irreducible surface which is the base locus of $\left|\mathcal{I}_{C, M}(2)\right|$. We conclude as in step (d2.3.1).

Proof of Theorem 1: We just proved Theorem 1 for all $C \in M_{d, g}$ spanning a hyperplane of $\mathbb{P}^{5}$, and so we completed the proof of Theorem 1

\section{Curves in a 3-space}

In this section we assume that $C$ spans a 3-dimensional linear subspace $U \subset \mathbb{P}^{5}$. We prove Proposition 1 and give a few results useful to extend it to higher degrees and higher genera. The Hilbert scheme $M_{d, g}^{\prime}(U)$ of all non-special curves of degree $d$ and genus $g$ of $U$ is smooth and irreducible of dimension $4 d$. Since the Grassmannian $G(3,5)$ of all 3-dimensional linear subspaces of $\mathbb{P}^{5}$ has dimension 8 , to exclude these curves $C$ it is sufficient to exclude the ones with $h^{1}\left(\mathcal{I}_{C}(7)\right) \geq$ $2 d+2-2 g-8$. However, for a general $W \in \mathcal{W}$ and a general $U \in G(3,5)$ the surface $W \cap U$ is a general surface of degree 7 and hence any curve of degree $\leq 16$ on it is a complete intersection by a theorem of Max Noether. Hence to exclude all $C \in M_{d, g}$ spanning some $U \in G(3,5)$ it is sufficient to exclude the ones with $h^{1}\left(\mathcal{I}_{C}(7)\right) \geq 2 d-2 g-5$. Fix a general $X \in M_{d, g}^{\prime}(U)$. Since $X$ has maximal rank ([2]) and $7 d+1-g \leq 120=\left(\begin{array}{c}10 \\ 3\end{array}\right)$, we have $h^{1}\left(\mathcal{I}_{X}(7)\right)=h^{1}\left(U, \mathcal{I}_{X, U}(7)\right)=0$ and so $h^{0}\left(\mathcal{I}_{X}(7)\right)=\left(\begin{array}{c}12 \\ 5\end{array}\right)-7 d-1+g$. Since $\operatorname{dim}\left(M_{d, g}^{\prime}(U)\right)=4 d, \mathbb{P}^{5}$ has $\infty^{8}$ 3-dimensional linear spaces and $7 d+1-g>4 d+8$, a general heptic hypersurface contains no curve $C \in M_{d, g}$ with the Hilbert function of $X$ in degree 1 and degree 7 . Hence we may increase by one these bounds and so we may assume $h^{1}\left(U, \mathcal{I}_{C, U}(7)\right) \geq 2 d-2 g-4$, i.e. for any $d, g$ to rule out the existence of any $C \in M_{d, g}$ with $\operatorname{dim}(\langle C\rangle)=3$ for a general heptic hypersurface it is sufficient to exclude all $C \in M_{d, g}^{\prime}(U)$ with $h^{1}\left(U, \mathcal{I}_{C, U}(7)\right) \geq 2 d-2 g-4$. By [13] we may assume $d \geq 11$. Hence $11 \leq d \leq 16$ and $1 \leq g \leq 3$. Let $\alpha$ or $\alpha(C)$ be the minimal degree of a surface of $U$ containing $C$. Since $C$ is non-degenerate, we have $\alpha \geq 2$. Since $7 \cdot 16+1-g<120=\left(\begin{array}{c}10 \\ 3\end{array}\right)$, we have $\alpha \leq 7$.

Lemma 22. We have $\alpha \neq 2$.

Proof. Assume $\alpha=2$. Let $Q \subset U$ be a quadric containing $C$. If $Q$ is a quadric cone, then $C$ is arithmetically Cohen-Macaulay ([16, V, Ex. 2.9]), because $C$ is smooth, and in particular $h^{1}\left(\mathcal{I}_{C}(7)\right)=0$, a contradiction. Hence $Q$ is a smooth quadric. Write $C \in\left|\mathcal{O}_{Q}(u, v)\right|$ with, say, $u \leq v$. Since $g>0$, we have $u>1$. Since $h^{1}\left(\mathcal{O}_{C}(1)\right)=0$, we have $u \leq 2$. Since $1 \leq g \leq 3$, we get $u=2$ and $v=g+1$. In all cases we have $h^{1}\left(\mathcal{I}_{C}(7)\right)=0$, a contradiction. below.

The following lemma is a particular case of step (b2) of Remark 5 proved 
Lemma 23. Fix $C \in M_{d, g}^{\prime}(U), d \leq 16$. Let $H \subset U$ be a general hyperplane. We have $h^{1}\left(H, \mathcal{I}_{\mathrm{C} \cap H, H}(t)\right)=0$ for all $t \geq 5, h^{1}\left(H, \mathcal{I}_{C \cap H, H}(4)\right)=0$ if $d \leq 12$ and $h^{1}\left(H, \mathcal{I}_{\mathrm{C} \cap H, H}(4)\right) \leq 1$ if $d \in\{13,14\}$.

Remark 5. Let $H \subset U$ be a general plane. The set $Z:=C \cap H$ is formed by $d$ points of $H$ in uniform position and spanning $H$.

(a) In particular $Z$ is in linearly general position and hence $h^{1}\left(H, \mathcal{I}_{Z, H}(t)\right)=$ 0 for all $t$ with $2 t \geq d-1$ ([10, Theorem 3.2]). If $d \geq 2 t+2$ we get $h^{1}\left(H, \mathcal{I}_{Z, H}(t)\right) \leq$ $d-2 t-1$.

(b) Now assume $2 t \leq d-2$, but $\left(\begin{array}{c}t+2 \\ 2\end{array}\right) \geq d$, i.e., if $d=16$ assume $5 \leq t \leq 7$, while if $11 \leq d \leq 15$ assume $4 \leq t \leq\lfloor(d-2) / 2\rfloor$. Assume $h^{1}\left(H, \mathcal{I}_{Z, H}(t)\right)>0$. We get $h^{0}\left(H, \mathcal{I}_{Z, H}(t)\right)>0$. Since $C \cap H$ is in uniform position (or by a monodromy argument) and $d>t$, either a general $D \in\left|\mathcal{I}_{Z, H}(t)\right|$ is irreducible or the base locus of $\left|\mathcal{I}_{Z, H}(t)\right|$ contains an irreducible curve $T \supset Z$ and $D=c T$ for some integer $c \geq 2$. The latter case does not occur if $t$ is a prime, because $Z$ spans $H$.

(b1) Assume $D=c T$ with $c \geq 2$. We get $\left|\mathcal{I}_{Z, H}(t)\right|=\{c T\}$ and hence $h^{0}\left(H, \mathcal{I}_{Z, H}(t)\right)=1$. Therefore $h^{1}\left(H, \mathcal{I}_{Z, H}(t)\right)=d+1-\left(\begin{array}{c}t+2 \\ 2\end{array}\right)$. Since $d \leq\left(\begin{array}{c}t+2 \\ 2\end{array}\right)$, we get $d=\left(\begin{array}{c}t+2 \\ 2\end{array}\right)$ and $h^{1}\left(H, \mathcal{I}_{Z, H}(t)\right)=1$. Since $Z$ spans $H, t$ is not a prime. Since $d \leq 16$, we get $t<6$. Hence $t=4, c=2, \operatorname{deg}(T)=2$ and $d=15$. Since $T$ is an integral conic (i.e. a smooth conic), we get $h^{1}\left(H, \mathcal{I}_{Z, H}(y)\right)=0$ for all $y \geq 7$. Let $K \subset U$ be a degree $\alpha$ surface containing $C$. Recall that $\alpha \leq 7$. Since $d=15>2 \alpha$, Bezout theorem gives $T \subset K$. Varying $H$ we see that $K$ contains a 3-dimensional family of conics and hence it is a projection of a Veronese surface. Hence $\alpha \leq 4$. Since $C$ has odd degree $K$ must be an inner projection of the Veronese surface and hence $\alpha=3$. Since $C$ is smooth, we see that $C$ is isomorphic to a plane curve of degree $\geq 8$, contradicting the assumption $g \leq 3$.

(b2) Assume that $D$ is irreducible. Since $h^{i}\left(\mathcal{O}_{H}\right)=0, i=1,2$, then we have $h^{1}\left(H, \mathcal{I}_{Z, H}(t)\right)=h^{1}\left(D, \mathcal{I}_{Z, D}(t)\right)$. Since $\omega_{D} \cong \mathcal{O}_{D}(t-3)$, we get $d \geq 3 t$. If $d=3 t$ we also get that $Z$ is the complete intersection of $D$ with a plane cubic; since $h^{1}\left(U, \mathcal{I}_{C, U}(7)\right)>0,[33$, Theorem 0.1$]$ gives that $Z$ is contained in a smooth conic, contradicting the inequality $d>2 t$ and the irreducibility of $D$. Now assume $d \geq 3 t+1$. Since $h^{1}\left(D, \mathcal{I}_{Z, D}(t)\right)>0, \mathcal{I}_{Z, D}(t)$ is a subsheaf of $\omega_{D} \cong \mathcal{O}_{D}(t-3)$ and hence there is $Z^{\prime} \subset Z$ such that $\operatorname{deg}\left(Z^{\prime}\right)=3 t$ and $Z^{\prime}$ is the complete intersection of $D$ and and a cubic curve. We have $h^{1}\left(H, \mathcal{I}_{Z, H}(t)\right)=$ $h^{1}\left(D, \mathcal{I}_{Z, D}(t)\right)$ and $h^{1}\left(H, \mathcal{I}_{Z^{\prime}, H}(t)\right)=h^{1}\left(D, \mathcal{I}_{Z^{\prime}, D}(t)\right)=h^{1}\left(D, \mathcal{O}_{D}(t-3)\right)=1$. Since $\mathcal{O}_{D}(t-3)$ is very ample, we have $h^{0}\left(D, \mathcal{I}_{E, D}(t)\right)=h^{0}\left(D, \mathcal{I}_{Z^{\prime}, D}(t)\right)-\operatorname{deg}(E)$ (i.e. $h^{1}\left(D, \mathcal{I}_{E, D}(t)\right)=h^{1}\left(D, \mathcal{I}_{Z^{\prime}, D}(t)\right)$ for every $E \supset Z^{\prime}$ with $\operatorname{deg}(E)-\operatorname{deg}\left(Z^{\prime}\right) \leq$ 2. Hence if $h^{1}\left(H, \mathcal{I}_{Z, H}(t)\right)>0$, then $d \geq 3 t+1$ and if $d \leq 3 t+2$, then $h^{1}\left(H, \mathcal{I}_{Z, H}(t)\right) \leq 1$.

\subsection{Proof of Proposition 1}

In this subsection we take $g=1$ and $d \leq 14$.

Lemma 24. Fix a zero-dimensional scheme $Z \subset U$ and set $b:=\operatorname{deg}(Z)$. Let $E_{Z}$ be the set of all $C \in M_{d, 1}^{\prime}(U)$ such that $C \supset Z$. If $d \geq b$, then every irreducible component of $E_{Z}$ has dimension $4 d-2 b$. 
Proof. Fix $C \in E_{Z}$. It is sufficient to prove that $h^{1}\left(N_{C, U}(-Z)\right)=0$ ([27, Theorem 1.5]). Since $N_{C}$ is a quotient of $\mathcal{O}_{C}(1)^{\oplus 4}$, we are done if $h^{1}\left(\mathcal{O}_{C}(1)(-Z)\right)=0$. Since $d \geq b$ and $\omega_{C} \cong \mathcal{O}_{Z}$, this is the case, unless $d=b$ and $\mathcal{O}_{C}(1) \cong \mathcal{O}_{C}(Z)$. By duality we only need to exclude the existence of a non-zero map $N_{C} \rightarrow \mathcal{O}_{C}(1)$. Assume that this is the case. The restriction to $C$ of Euler's sequence of $T \mathbb{P}^{3}$ gives a non-zero map $\mathcal{O}_{C}(1)^{\oplus 4} \rightarrow \mathcal{O}_{C}(1)$. This map gives the equation of a hyperplane of $U$ containing $C$, a contradiction.

Lemma 25. A general $W \in \mathcal{W}$ contains no $C \in M_{d, 1}, d \leq 14$, such that there is $U \in G(3,5)$ with $C \in M_{d, 1}^{\prime}(U), \operatorname{deg}(R \cap C) \leq 7$ for each line $R$ and $\operatorname{deg}(C \cap D) \leq 13$ for each conic $D$.

Proof. Fix $C \in M_{d, 1}^{\prime}(U)$ such that $\operatorname{deg}(R \cap C) \leq 7$ for each line $R$ and $\operatorname{deg}(R \cap D) \leq 13$ for each conic $D$. By Lemmas 1 and 23 we have $h^{1}\left(U, \mathcal{I}_{C, U}(5)\right) \geq$ $h^{1}\left(U, \mathcal{I}_{C, U}(6)\right)+3 \geq h^{1}\left(U, \mathcal{I}_{C, U}(7)\right)+6 \geq 2 d$. By Lemma 23 we have $h^{1}\left(U, \mathcal{I}_{C, U}(3)\right) \geq h^{1}\left(U, \mathcal{I}_{C, U}(7)\right)-\epsilon \geq 2 d-\epsilon$ with $\epsilon=0$ if $d \leq 12$ and $\epsilon=1$ if $d \in\{13,14\}$, i.e. $h^{0}\left(U, \mathcal{I}_{C, U}(3)\right) \geq 20-d-\epsilon \geq 2$. Since $\alpha>2$ (Lemma 22), $C$ is contained in the intersection of 2 integral cubic surfaces and so $d \leq 9$, a contradiction.

Lemma 26. A general $W \in \mathcal{W}$ contains no $C \in M_{d, 1}, d \leq 14$, such that there is $U \in G(3,5)$ with $C \in M_{d, 1}^{\prime}(U)$ and a conic $D$ with $\operatorname{deg}(D \cap C) \geq 14$.

Proof. Take $C \in M_{d, 1}, d \leq 14$, such that there is $U \in G(3,5)$ with $C \in M_{d, 1}^{\prime}(U)$ and a conic $D$ with $\operatorname{deg}(D \cap C) \geq 14$. We have $d=14$ and $\operatorname{deg}(D \cap C)=14$. By Lemma 25 we may assume that $\operatorname{deg}(R \cap C) \leq 6$ for every line $R$. Hence $D$ is a smooth conic and so it has $\infty^{14}$ degree 14 subschemes. Since $\operatorname{dim}(G(3,5))=8$, $U$ has $\infty^{3}$ planes and each plane has $\infty^{5}$ conics, to prove the lemma (quoting Lemma 24) it is sufficient to exclude all $C \in M_{14,1}$ spanning a 3-space $U$ and with $h^{1}\left(U, \mathcal{I}_{C, U}(7)\right) \geq 6 d+2-2 g-4 d-8+28-14-8=2 d-2$. By Lemma 23 we have $h^{1}\left(U, \mathcal{I}_{C, U}(3)\right) \geq h^{1}\left(U, \mathcal{I}_{C, U}(7)\right)-\epsilon \geq 2 d-2-\epsilon$ with $\epsilon=0$ if $d \leq 12$ and $\epsilon=1$ if $d \in\{13,14\}$, i.e. $h^{0}\left(U, \mathcal{I}_{C, U}(3)\right) \geq 18-d-\epsilon \geq 2$ and so Bezout gives $d \leq 9$, a contradiction.

Lemma 27. A general $W \in \mathcal{W}$ contains no $C \in M_{d, 1}, d \leq 14$, such that there is $U \in G(3,5)$ with $C \in M_{d, 1}^{\prime}(U)$ and a line $R$ with $\operatorname{deg}(R \cap C) \geq 8$ for some line $R$.

Proof. Fix $U$ and $C \in M_{d, 1}^{\prime}(U)$. By Lemma 26 we may assume $\operatorname{deg}(D \cap C) \leq 13$ for each conic $D$. Let $R \subset U$ be a line such that $b:=\operatorname{deg}(C \cap R)$ is maximal. First assume $b \geq 9$. Fix $Z \subset R \cap C$ with $\operatorname{deg}(Z)=9$. Since $\operatorname{dim}(G(3,5))=8, U$ has $\infty^{4}$ lines and each line has $\infty^{9}$ subschemes of degree 9, Lemma 24 shows that it is sufficient to exclude the curves $C$ with $h^{1}\left(U, \mathcal{I}_{C, U}(7)\right) \geq 2 d-8+18-9-4=$ $2 d-3$. By Lemma 23 we have $h^{1}\left(U, \mathcal{I}_{C, U}(3)\right) \geq 2 d-3-\epsilon$ with $\epsilon=0$ if $d \leq 12$ and $\epsilon=1$ if $d \in\{13,14\}$, i.e. $h^{0}\left(U, \mathcal{I}_{C, U}(3)\right) \geq 17-d-\epsilon \geq 2$ and so Bezout gives $d \leq 9$, a contradiction. Now assume $b=8$. As above we see that it is sufficient to exclude all $C \in M_{d, 1}^{\prime}(U)$ with $h^{1}\left(U, \mathcal{I}_{C, U}(7)\right) \geq 2 d-4$. By Lemmas 1 and 23 we have $h^{1}\left(U, \mathcal{I}_{C, U}(6)\right) \geq 2 d-1$ and hence $h^{0}\left(U, \mathcal{I}_{C, U}(3)\right) \geq 4$.

Proof of Proposition 1. Lemmas 25, 26 and 27 prove Proposition 1. 


\section{References}

[1] E. Ballico and Ph. Ellia, On postulation of curves in $\mathbb{P}^{4}$, Math. Z. 188, 1985), no. 2, 215-223.

[2] E. Ballico and Ph. Ellia, The maximal rank conjecture for non-special curves in $\mathbf{P}^{3}$, Invent. Math. 79 (1985), 541-555.

[3] E. Ballico and Ph. Ellia, The maximal rank conjecture for non-special curves in $\mathbb{P}^{n}$, Math. Zeit. 196 (1987), 585-599.

[4] A. Bernardi, A. Gimigliano, and M. Idà, Computing symmetric rank for symmetric tensors, J. Symbolic Comput. 46 (2011), no. 1, 34-53.

[5] H. Clemens, Some results about Abel-Jacobi mappings, Topics in transcendental algebraic geometry (Princeton, N.J., 1981/1982), 289-304, Ann. of Math. Stud., 106, Princeton Univ. Press, Princeton, NJ, 1984.

[6] E. Cotterill, Rational curves of degree 11 on a general quintic 3-fold, Quart. J. Math. 63 (2012), 539-568.

[7] E. Cotterill, Rational curves of degree 16 on a general heptic fourfold, J. Pure Appl. Algebra 218 (2014), 121-129.

[8] E. Cotterill, L. Feital and R. V. Martins, Singular rational curves in $\mathbb{P}^{n}$ via semigroups, arXiv:1511.08515.

[9] D'Almeida, Courbes rationnelles de degré 11 sur une hypersurface quintique générale de $\mathbb{P}^{4}$, Bull. Sci. math. 136 (2012), 899-903.

[10] D. Eisenbud and J. Harris, Finite projective schemes in linearly general position, J. Algebraic Geom. 1 (1992), no. 1, 15-30.

[11] Ph. Ellia and Ch. Peskine, Groupes de points de $\mathbf{P}^{2}$ : caractère et position uniforme, in: Algebraic geometry (L' Aquila, 1988), 111-116, Lecture Notes in Math., 1417, Springer, Berlin, 1990.

[12] H. Flenner, L. O'Carroll, and W. Vogel, Joins and intersections, Springer Monographs in Mathematics, Springer-Verlag, Berlin, 1999.

[13] L. Gruson, R. Lazarsfeld and Ch. Peskine, On a theorem of Castelnuovo, and the equations defining space curves, Invent. Math. 72 (1983), 491-506.

[14] G. Hana and T. Johnsen, Rational curves on a general heptic fourfold, Bull. Belg. Math. Soc., Simon Stevin 16 (2009), 861-885.

[15] J. Harris, with the collaboration of D. Eisenbud, Curves in Projective Space, Les Presses de l'Université de Montréal, Montréal, 1982.

[16] R. Hartshorne, Algebraic Geometry, Springer-Verlag, Berlin-HeidelbergNew York, 1977. 
[17] C. Huneke and B. Ulrich, General hyperplane sections of algebraic varieties, J. Algebraic Geom. 2 (1993), no. 3, 487-505.

[18] P. Jahnke, T. Peternell, and I. Radloff, Some recent developments in the classification theory of higher dimensional manifolds, Global aspects of complex geometry, 311-357, Springer, Berlin, 2006.

[19] T. Johnsen and S. Kleiman, Rational curves of degree at most 9 on a general quintic threefold, Comm. Algebra 24 (1996), 2721-2753.

[20] T. Johnsen and S. Kleiman, Toward Clemens' Conjecture in degrees between 10 and 24, Serdica Math. J. 23 (1997), 131-142.

[21] T. Johnsen and A. L. Knutsen, Rational curves in Calabi-Yau threefolds. Special issue in honor of Steven L. Kleiman. Comm. Algebra 31 (2003), no. 8, 3917-3953.

[22] S. Katz, On the finiteness of rational curves on quintic threefolds, Compositio Math. 60 (1986), 151-162.

[23] A. L. Knutsen, On isolated smooth curves of low genera in Calabi-Yau complete intersection threefolds, Trans. Amer. Math. Soc. 364 (2012), no. 10, 5243-5264.

[24] A. L. Knutsen, Smooth, isolated curves in families of Calabi-Yau threefolds in homogeneous spaces, J. Korean Math. Soc. 50 (2013), no. 5, 1033-1050.

[25] N. Mohan Kumar, A. P. Rao and G. V. Ravindra, On codimension two subvarieties in hypersurfaces, Motives and algebraic cycles, 167-174, Fields Inst. Commun., 56, Amer. Math. Soc., Providence, RI, 2009

[26] K. Oguiso, Two remarks on Calabi-Yau threefolds, J. Reine Angew. Math. 452 (1994), 153-161.

[27] D. Perrin, Courbes passant par $m$ points généraux de $\mathbb{P}^{3}$, Bull. Soc. Math. France, Mémoire 28/29 (1987).

[28] D. Shin, Rational curves on general hypersurfaces of degree 7 in $\mathbb{P}^{7}$, Osaka Journal of Mathematics 44 (2007), no. 1, 1-10.

[29] C. Voisin, Sur une conjecture de Griffiths et Harris, Algebraic curves and projective geometry (Trento, 1988), 270-275, Lecture Notes in Math., 1389, Springer, Berlin, 1989.

[30] C. Voisin, On a conjecture of Clemens on rational curves on hypersurfaces, J. Differential Geometry 44 (1996), no. 1, 200-213.

[31] C. Voisin. Correction to "On a conjecture of Clemens on rational curves on hypersurfaces, J. Differential Geometry 49 (1998), 601-611.

[32] C. Voisin, On some problems of Kobayashi and Lang, in Current developments in Mathematics, 2003, pp. 53-125, Int. Press, Somerville, MA, 2003. 
[33] K. Yanagawa, A characterization of integral curves with Gorenstein hyperplane sections, Proc. Amer. Math. Soc. 124 (1996), no. 5, 1379-1384.

Dept. of Mathematics

University of Trento

38123 Povo (TN), Italy

email: ballico@science.unitn.it 